\title{
General Covariance in Quantum Gravity at a Lifshitz Point
}

\author{
Petr Hořava ${ }^{a, b, c}$ and Charles M. Melby-Thompson ${ }^{a, b}$ \\ ${ }^{a}$ Berkeley Center for Theoretical Physics and Department of Physics \\ University of California, Berkeley, CA, 94720-7300, USA \\ ${ }^{b}$ Theoretical Physics Group, Lawrence Berkeley National Laboratory \\ Berkeley, CA 94720-8162, USA \\ ${ }^{c}$ Institute for the Physics and Mathematics of the Universe \\ University of Tokyo, Kashiwa 277-8568, Japan
}

\begin{abstract}
In the minimal formulation of gravity with Lifshitz-type anisotropic scaling, the gauge symmetries of the system are foliation-preserving diffeomorphisms of spacetime. Consequently, compared to general relativity, the spectrum contains an extra scalar graviton polarization. Here we investigate the possibility of extending the gauge group by a local $U(1)$ symmetry to "nonrelativistic general covariance." This extended gauge symmetry eliminates the scalar graviton, and forces the coupling constant $\lambda$ in the kinetic term of the minimal formulation to take its relativistic value, $\lambda=1$. The resulting theory exhibits anisotropic scaling at short distances, and reproduces many features of general relativity at long distances.
\end{abstract}




\section{Contents}

1. Introduction 1

1.1 General covariance

1.2 The minimal theory of gravity with anisotropic scaling

1.3 Comments on the nonprojectable case 8

2. Global $U(1)_{\Sigma}$ Symmetry in the Minimal Theory at $\lambda=1$

2.1 Symmetries in the linearized approximation around flat spacetime 12

2.2 The nonlinear theory

3. Gauging the $U(1)_{\Sigma}$ Symmetry: First Examples 16

3.1 Geometric interpretation of the $U(1)$ symmetry

3.1.1 The gauge field $A$ and the Newton potential

3.1.2 Extending the $1 / c$ expansion 18

3.2 Generally covariant nonrelativistic gravity in $2+1$ dimensions

3.3 Self-interacting Abelian gravity 21

4. General Covariance at a Lifshitz Point 22

4.1 Repairing the global $U(1)_{\Sigma}$ symmetry 23

4.1.1 The Newton prepotential 23

4.1.2 Relevant deformations 23

4.1.3 Linearized theory with global $U(1)_{\Sigma}$ around flat spacetime 24

4.2 Gauging the $U(1)_{\Sigma}$ symmetry 26

4.2.1 Hamiltonian formulation 27

4.2.2 Linearization around detailed balance 30

5. Conclusions

5.1 Static compact-object solutions

5.2 Lorentz symmetry 35

5.3 Cosmological solutions 37

\section{Introduction}

The idea of gravity with anisotropic scaling [1-3] has attracted a lot of attention recently. There are two, somewhat distinct, motivations for developing this approach to gravity. The first is driven by the long-standing search for a theoretical framework in which the classical 
theory of gravity is reconciled with the laws of quantum mechanics. A successful outcome of this search would result in a mathematically self-consistent framework for quantum gravity, not necessarily subjected to experimental tests. Examples already exist - the ten-dimensional supersymmetric vacua of string theory belong to this category. The second motivation comes from a goal which is more narrow, and also much more ambitious: To find, within such a selfconsistent quantum gravity framework, a theory that reproduces the observed gravitational phenomena in our universe of $3+1$ macroscopic dimensions.

Both of these motivations are relevant for the development of gravity with anisotropic scaling. For a large class of possible applications, it does not matter whether or not the theory matches general relativity at long distances, or conforms to the available experimental tests of gravity in $3+1$ dimensions. A mathematically consistent quantum gravity which lacks this phenomenological matching can still be useful in the context of AdS/CFT correspondence, and produce novel gravity duals for a broader class of field theories, of interest for example in condensed matter applications. It can also have interesting mathematical implications, given the close connection between the theory formulated in $[1,2]$ and the mathematical theory of the Ricci flow on Riemannian manifolds.

However, explaining the observed features of gravity in our universe of $3+1$ dimensions is still perhaps the leading motivation for developing a quantum theory of gravity. Therefore, it makes sense to ask how close we can get, in the new framework of gravity with anisotropic scaling, to reproducing general relativity in the range of scales where the laws of gravity have been experimentally tested.

The comparison to general relativity is facilitated by the fact that in the framework proposed in [1-3], gravity is also described simply as a field theory of the dynamical metric on spacetime. Unlike in general relativity, however, the spacetime manifold $M$ (which we take to be of a general dimension $D+1$ ) is equipped with a preferred structure of a codimensionone foliation $\mathcal{F}$ by slices of constant time, $\Sigma(t) .{ }^{1}$ In the minimal realization of the theory, reviewed in Section 1.2, the gauge symmetries of the system are the foliation-preserving diffeomorphisms $\operatorname{Diff}(M, \mathcal{F})$. Since this symmetry contains one less gauge invariance per spacetime point compared to the full spacetime diffeomorphisms $\operatorname{Diff}(M)$, the spectrum of the linearized theory around flat spacetime contains one additional, scalar polarization of the graviton.

At short distances, the anisotropy between space and time is measured by a nontrivial dynamical critical exponent $z>1$, leading to an improved ultraviolet behavior of the theory. At long distances, on the other hand, the theory is driven to an infrared regime where it shares many features with general relativity. First of all, under the influence of relevant terms in the classical action, the scaling becomes naturally isotropic, with the relativistic value of $z=1$. Moreover, the lowest-dimension terms that dominate the action in the infrared are exactly those that appear in the ADM decomposition [4] of the Einstein-Hilbert action: The scalar

\footnotetext{
${ }^{1}$ For simplicity, we will assume throughout this paper that the leaves $\Sigma(t)$ of this foliation all have the same topology of a $D$-dimensional manifold $\Sigma$.
} 
curvature term, which sets the value of the effective Newton constant, and the cosmological constant term.

Thus, in the low-energy regime, the action of the minimal theory with anisotropic scaling looks very similar to that of general relativity. However, this similarity has its limits, and the theories are clearly different even in the infrared. The differences can be understood in three related ways: As a difference in gauge symmetries, a difference in the graviton spectrum, and a difference in the number of independent coupling constants. First, in the theory with anisotropic scaling, the gauge symmetry is reduced to $\operatorname{Diff}(M, \mathcal{F})$, and the theory propagates an extra scalar polarization of the graviton. In addition, the kinetic term in the action allows for an additional coupling $\lambda$, which is undetermined by any symmetry of the minimal theory, and therefore expected to run with the scale in the quantum theory. In general relativity, the spacetime diffeomorphism symmetries force $\lambda=1$ and protect this value from quantum corrections. Stringent experimental limits on the value of $\lambda$ have been advocated in the literature $[5,6]$, suggesting that at least in this class of models, it must be very near its relativistic value $\lambda=1$. However, in the regime near $\lambda=1$, difficulties with the dynamics of the additional scalar graviton have been pointed out (see, for example, [7-9]).

In order to get closer to general relativity, it is tempting to focus on the structure of gauge symmetries. However, this needs to be done cautiously, keeping in mind that gauge symmetries are just convenient redundancies in the description of a physical system, and therefore to some extent in the eye of the beholder. The more physical perspective is to focus on the spectrum of propagating degrees of freedom. ${ }^{2}$ Thus, we will be interested in finding an extension of the gauge symmetry that will turn the extra, scalar polarization of the graviton into a gauge artifact. A second option would be to find a mechanism for generating a finite mass gap for the scalar graviton - in this paper, we concentrate on the first possibility.

We will find such an extended gauge symmetry, with as many generators per spacetime point as in general relativity. This gauge symmetry can be viewed as representing "nonrelativistic general covariance" in gravity with anisotropic scaling. The extended symmetry eliminates the scalar polarization of the graviton from the spectrum. As a bonus, we find that the extended gauge symmetry requires $\lambda=1$, thereby reducing the kinetic term to coincide with that of general relativity. It is in fact important that our entire construction depends only on the form of the kinetic term, and therefore does not restrict the form of the potential term in the action. Hence, at short distances, the covariant theory can exhibit the same improved ultraviolet behavior associated with $z>1$ in the minimal theory of $[1,2]$.

\footnotetext{
${ }^{2}$ Of course, one can make the theory diffeomorphism invariant in a trivial way, by integrating in the gauge invariance without changing the number of physical degrees of freedom. This leads to a theory which is formally generally covariant, but effectively equivalent to the original model. Perhaps any sensible theory can be "parametrized" in this way [10], and formally rewritten as a generally covariant theory. This process of covariantizing a given theory is very closely connected to the Stückelberg mechanism used prominently in particle physics (see, e.g., [11] for a review). It has been applied to the models of gravity with anisotropic scaling $[1,2]$ in $[7,12,13]$. In this paper, instead, we are interested in the nontrivial extension of gauge symmetry which actually reduces the number of physical degrees of freedom. Investigating how the resulting theory then responds to the Stückelberg trick is an interesting question, beyond the scope of this paper.
} 
In this paper, our perspective is that of (effective) quantum field theory, but we restrict our analysis to the leading tree-level, or classical, approximation. Quantum corrections are expected to modify the scaling behavior of our models, but they are beyond the scope of this paper. In addition, our analysis will be strictly local: We freely integrate by parts and ignore total derivative terms. Boundary terms play a notoriously central role in relativistic gravity; it will be important to extend our analysis to include their precise structure and clarify their role in theories of gravity with anisotropic scaling. These are among the many interesting issues left for future work.

The main result of the paper is the construction of the generally covariant gravity with anisotropic scaling, which we present in Section 4 . Sections 1 2 2 and 3 prepare the ground for a better understanding of the main results, and explore a few additional issues of interest.

\subsection{General covariance}

In order to explain what exactly we mean by "general covariance," we first consider two theories - general relativity, and the ultralocal theory of gravity [14,15] - and illustrate our point using the Hamiltonian formulation of these two theories.

In fact, throughout this paper we will often resort to the Hamiltonian formalism, ${ }^{3}$ for a number of reasons. First of all, the time versus space split of the Hamiltonian formalism is particularly natural for gravity with anisotropic scaling. More importantly, the technology available in the Hamiltonian formalism allows us to get a precise count of the number of propagating degrees of freedom, and offers a better insight into the structure of the gauge symmetries of the theory. Indeed, one of the advantages of the Hamiltonian formulation is that one does not have to specify the gauge symmetries a priori. Instead, the structure of the Hamiltonian constraints provides an essentially algorithmic way in which the correct gauge symmetry structure is determined automatically [10]. In the process, the consistency of the equations of motion is tied to the closure of the constraint algebra and the preservation of the constraints under the time evolution. Once the full system of constraints has been determined, the constraints are separated into first-class (whose commutators with other constraints vanish on the constraint surface) and second-class (whose commutators define a nondegenerate symplectic form). As an additional benefit, after determining the numbers $\mathcal{C}_{1}$ of first-class and $\mathcal{C}_{2}$ of second-class constraints, the number of degrees of freedom $\mathcal{N}$ can be reliably evaluated by the standard formula [10]

$$
\mathcal{N}=\frac{1}{2}\left(\operatorname{dim} \mathcal{P}-2 \mathcal{C}_{1}-\mathcal{C}_{2}\right)
$$

where $\operatorname{dim} \mathcal{P}$ is the number of fields in the canonical formulation (i.e., the dimension of phase space). In local field theory, this formula can be interpreted per spacetime point, giving the number of local degrees of freedom. We will use this formula repeatedly throughout the paper.

\footnotetext{
${ }^{3}$ For the canonical reference on Hamiltonian systems with constraints, see [10].
} 
In canonical general relativity $[4,16,17]$ on a spacetime manifold $M$ with $D+1$ coordinates $\left(x^{i}, t\right)$, the algebra of constraints contains the "superhamiltonian" $\mathcal{H}_{\perp}(\mathbf{x})$ and the "supermomentum" $\mathcal{H}_{i}(\mathbf{x})$, and the total Hamiltonian is just a sum of constraints:

$$
H=\int d^{D} \mathbf{x}\left(N \mathcal{H}_{\perp}+N^{i} \mathcal{H}_{i}\right)
$$

$N$ and $N^{i}$ are the lapse and shift variables of the metric, and $\mathcal{H}_{\perp}$ and $\mathcal{H}_{i}$ are functions of the spatial components $g_{i j}$ of the metric and their canonically conjugate momenta $\pi^{i j}$. Since the constraints are all first-class, they generate gauge symmetries, whose generators are

$$
\mathcal{H}\left(\xi^{i}\right) \equiv \int d^{D} \mathbf{x} \xi^{i}(\mathbf{x}, t) \mathcal{H}_{i}(\mathbf{x}, t), \quad \mathcal{H}_{\perp}\left(\xi^{0}\right) \equiv \int d^{D} \mathbf{x} \xi^{0}(\mathbf{x}, t) \mathcal{H}_{\perp}(\mathbf{x}, t) .
$$

Their commutation relations are well-understood, even though they do not quite yield the naively expected spacetime diffeomorphism algebra. True, the commutator of two $\mathcal{H}_{i}$ 's

$$
\left[\mathcal{H}\left(\xi^{i}\right), \mathcal{H}\left(\zeta^{j}\right)\right]=\mathcal{H}\left(\xi^{k} \partial_{k} \zeta^{i}-\zeta^{k} \partial_{k} \xi^{i}\right)
$$

reproduces the algebra of spatial diffeomorphisms Diff $(\Sigma)$, and

$$
\left[\mathcal{H}\left(\xi^{i}\right), \mathcal{H}_{\perp}\left(\zeta^{0}\right)\right]=\mathcal{H}_{\perp}\left(\xi^{k} \partial_{k} \zeta^{0}\right)
$$

just states that $\mathcal{H}_{\perp}$ transforms correctly under $\operatorname{Diff}(\Sigma)$. However, the commutator of $\mathcal{H}_{\perp}$ with itself gives a field-dependent result,

$$
\left[\mathcal{H}_{\perp}\left(\xi^{0}\right), \mathcal{H}_{\perp}\left(\zeta^{0}\right)\right]=-\sigma \mathcal{H}\left(g^{i j}\left(\xi^{0} \partial_{j} \zeta^{0}-\zeta^{0} \partial_{j} \xi^{0}\right)\right)
$$

Here $\sigma$ denotes the signature of spacetime: $\sigma=-1$ for general relativity in Minkowski signature. This generalized, Dirac algebra is the Hamiltonian manifestation of the original diffeomorphism symmetry (and general covariance) of general relativity, with $D+1$ gauge symmetries per spacetime point.

Our second example is the ultralocal theory of gravity, which results from dropping the spatial scalar curvature term $R$ in the action of general relativity. Of course, this step selects a preferred foliation $\mathcal{F}$ of spacetime, and therefore violates spacetime diffeomorphism invariance. One might naively assume that the symmetry is reduced to the foliation-preserving diffeomorphisms $\operatorname{Diff}(M, \mathcal{F})$. However, the analysis of Hamiltonian constraints reveals a surprising fact $[14,15]$ : The theory is still gauge invariant under as many gauge symmetries per spacetime point as general relativity. In contrast with general relativity, the ultralocal theory exhibits a contracted version of the Hamiltonian constraint algebra, with (1.6) replaced by its $\sigma \rightarrow 0$ limit:

$$
\left[\mathcal{H}_{\perp}\left(\xi^{0}\right), \mathcal{H}\left(\zeta^{0}\right)\right]=0
$$

while the remaining commutators (1.4) and (1.5) stay the same.

The theory is "generally covariant" - it has the same number $D+1$ of (nontrivial) local gauge symmetries as general relativity, even though the algebra in the $\sigma \rightarrow 0$ limit 
still preserves the preferred spacetime foliation structure. Effectively, the spatial diffeomorphism symmetries have been kept intact, but the time reparametrization symmetry has been linearized, and its algebra contracted to a local $U(1)$ gauge symmetry. ${ }^{4}$ Just as the Dirac algebra of Hamiltonian constraints (1.4), (1.5) and (1.6) in general relativity is associated with the Lagrangian symmetries described by the group of spacetime diffeomorphisms, Diff $(M)$, the Teitelboim-Henneaux algebra (1.4), (1.5) and (1.7) can be associated with a Lagrangian symmetry group which takes the form of a semi-direct product,

$$
U(1) \ltimes \operatorname{Diff}(M, \mathcal{F}) .
$$

It is natural to interpret (1.8) as the symmetry group of "nonrelativistic general covariance." This is the structure of gauge symmetries that we will try to implement in the case of gravity with anisotropic scaling for general values of $z$.

The restoration of general covariance characterized by (1.8) still maintains the special status of time, keeping it on a different footing from space. We view the fact that "time is different" as a virtue of this approach: Indeed, we are looking for possible concessions on the side of general relativity that would make it friendlier to the way in which time is treated in quantum mechanics, without changing too much of its elegant geometric nature.

\subsection{The minimal theory of gravity with anisotropic scaling}

Here we review the basics of the simplest version of quantum gravity with anisotropic scaling $[1,2]$.

We assume that the spacetime manifold $M$ is a differentiable manifold equipped with the extra structure of a preferred foliation $\mathcal{F}$ by codimension-one leaves $\Sigma$ of constant time. Our starting point is a theory whose only dynamical field is the spatial metric, represented in a coordinate system $\left(\mathbf{x} \equiv x^{i}, t\right)$ on $\Sigma$ by components $g_{i j}(\mathbf{x}, t)$. In a sense, this is the most "primitive" implementation of the idea of anisotropic scaling for gravity: The theory exhibits no (time-dependent) gauge symmetries, and consequently the spectrum will contain not just the tensor polarizations of the gravitons, but also the vector and scalar graviton modes.

The kinetic term is written using the generalized De Witt metric ${ }^{5}$

$$
G^{i j k \ell}=\frac{1}{2}\left(g^{i k} g^{j \ell}+g^{i \ell} g^{j k}\right)-\lambda g^{i j} g^{k \ell} .
$$

The parameter $\lambda$ is left undetermined by the symmetries of the theory, and plays the role of an additional coupling constant.

This "primitive" theory becomes more interesting when we make it gauge invariant under foliation-preserving diffeomorphisms $\operatorname{Diff}(M, \mathcal{F})$, whose generators are

$$
\delta t=f(t), \quad \delta x^{i}=\xi^{i}(t, \mathbf{x}) .
$$

\footnotetext{
${ }^{4}$ Throughout this paper, we use the rather loose notation common in high-energy physics, and refer to any one-dimensional Abelian symmetry group as $U(1)$, regardless of whether or not it is actually compact. Moreover, we use the same notation also for the infinite-dimensional, gauge version of the $U(1)$ symmetry.

${ }^{5} G^{i j k \ell}$ can be viewed as a metric on the space of symmetric 2 -tensors. As in [1,2], we will denote its inverse by $\mathcal{G}_{i j k \ell}$, to distinguish it from $G^{i j k \ell}$ with all four indices lowered via $g_{i j}$.
} 
The minimal multiplet of fields now contains $g_{i j}$ plus the lapse function $N$ and the shift vector $N_{i}$, which transform under $(1.10)$ as

$$
\begin{aligned}
\delta g_{i j} & =\partial_{i} \xi^{k} g_{j k}+\partial_{j} \xi^{k} g_{i k}+\xi^{k} \partial_{k} g_{i j}, \\
\delta N_{i} & =\partial_{i} \xi^{j} N_{j}+\xi^{j} \partial_{j} N_{i}+\dot{\xi}^{j} g_{i j}+\dot{f} N_{i}+f \dot{N}_{i}, \\
\delta N & =\xi^{j} \partial_{j} N+\dot{f} N+f \dot{N} .
\end{aligned}
$$

The lapse and shift $N$ and $N_{i}$, known from the ADM parametrization of the spacetime metric in general relativity [4], play the role of gauge fields of the foliation-preserving diffeomorphism symmetry. Indeed, (1.11) shows that $N$ (or, more precisely, $\log N$ ), and $N^{i}$ transform as gauge fields under the gauge transformations,

$$
\frac{\delta N}{N}=\dot{f}+\ldots, \quad \delta N^{i}=\dot{\xi}^{i}+\ldots
$$

(Here the "..." stand for the standard Lie-derivative terms in (1.11).) As a result, it is natural to assume that $N$ and $N_{i}$ inherit the same dependence on spacetime as the corresponding generators (1.10): While $N_{i}(t, \mathbf{x})$ is a spacetime field, $N(t)$ is only a function of time, constant along the spatial slices $\Sigma$. Making this assumption about the lapse function will lead to the minimal theory of gravity with anisotropic scaling.

The covariantization of the "primitive" theory is accomplished by replacing the spatial volume element with its covariant version,

$$
\sqrt{g} \rightarrow \sqrt{g} N
$$

and by trading the time derivative of the metric for the extrinsic curvature $K_{i j}$ of the leaves of the foliation $\mathcal{F}$,

$$
\dot{g}_{i j} \rightarrow 2 K_{i j} \equiv \frac{1}{N}\left(\dot{g}_{i j}-\nabla_{i} N_{j}-\nabla_{j} N_{i}\right) .
$$

In this fashion, we obtain the minimal realization of the idea of anisotropic scaling in gravity. This minimal theory is sometimes referred to as "projectable," because the spacetime metric assembled from the ingredients $g_{i j}, N_{i}$ and $N$ satisfies the axioms of a "projectable metric" on $(M, \mathcal{F})$, as defined in the geometric theory of foliations. The action of the minimal theory is

$$
S=\frac{2}{\kappa^{2}} \int d t d^{D} \mathbf{x} \sqrt{g} N\left(K_{i j} K^{i j}-\lambda K^{2}-\mathcal{V}\right),
$$

where $K=g^{i j} K_{i j}$. The potential term $\mathcal{V}$ is an arbitrary Diff $(\Sigma)$-invariant local scalar functional built out of the spatial metric, its Riemann tensor and the spatial covariant derivatives, without the use of time derivatives.

Around a free-field fixed point with dynamical exponent $z$, we will measure the scaling dimensions of fields in the units of the spatial momentum: $\left[\partial_{i}\right] \equiv 1$. In these units, the volume element in the action is of dimension $\left[d t d^{D} \mathbf{x}\right]=-z-D$, suggesting the natural scaling dimensions for the field multiplet,

$$
\left[g_{i j}\right]=0, \quad\left[N_{i}\right]=z-1, \quad[N]=0 .
$$


This scaling further implies that $\left[\kappa^{2}\right]=z-D$.

If we wish for the theory to be power-counting renormalizable, it is natural to start the analysis of possible terms appearing in $\mathcal{V}$ at short distances. Around a hypothetical Gaussian fixed point, power-counting renormalizability in $D+1$ dimensions requires $\mathcal{V}$ to be dominated by terms with $2 D$ spatial derivatives, implying in turn that the dynamical critical exponent should be equal to $z=D$. For example, in $3+1$ dimensions, there is a natural potential

$$
\mathcal{V}_{\mathrm{UV}}=w^{2} C_{i j} C^{i j}+\ldots
$$

where $w$ is a dimensionless coupling, and $C^{i j}=\varepsilon^{i k \ell} \nabla_{k}\left(R_{\ell}^{j}-\frac{1}{4} R \delta_{\ell}^{j}\right)$ is the Cotton tensor. In (1.17), we have indicated only the part of the potential that is dominant in the ultraviolet, with "..." denoting the relevant terms which contain fewer than six spatial derivatives and become important at longer distances.

Under the influence of the relevant terms, the theory will flow, until it is dominated at long distances by the most relevant terms. In this regime, it makes sense to reorganize the terms in $\mathcal{V}$ by focusing on those most dominant in the infrared:

$$
\mathcal{V}_{\mathrm{IR}}=-\mu^{2}(R-2 \Lambda)+\ldots
$$

Here $\mu$ and $\Lambda$ are dimensionful couplings of dimensions $[\mu]=z-1$ and $[\Lambda]=2$, and the "..." now denote all the terms containing composite operators of higher dimension compared to the displayed, most dominant infrared terms.

It is useful to note that the algebra of gauge symmetries $\operatorname{Diff}(M, \mathcal{F})$, and their action on the fields, can be obtained simply by taking a nonrelativistic reduction of the fully relativistic spacetime diffeomorphism symmetry and its action on the relativistic metric $g_{\mu \nu}$. As we will see in Section 3.1, a natural extension of this procedure to subleading terms in $1 / c$ leads to a natural geometric interpretation of the extended symmetries that are the focus of this paper.

\subsection{Comments on the nonprojectable case}

The minimal, projectable theory can be rewritten in the Hamiltonian formalism, with the Hamiltonian similar to (1.2),

$$
H=\int d^{D} \mathbf{x}\left(N \mathcal{H}_{0}+N^{i} \mathcal{H}_{i}\right)
$$

Here $\mathcal{H}_{0}$ and $\mathcal{H}_{i}$ are again functions of the spatial metric and its conjugate momenta. In fact, $\mathcal{H}_{i}$ takes the same form as in general relativity, $\mathcal{H}^{i}=-2 \nabla_{k} \pi^{i k}$, and $\mathcal{H}_{0}$ depends on the choice of $\mathcal{V}$. The main conceptual difference compared to general relativity stems from the fact that because $N(t)$ is independent of the spatial coordinates $x^{i}$, it only gives rise to the integral constraint $\int d^{D} \mathbf{x} \mathcal{H}_{0}=0$. Consequently, compared to general relativity, the number of first-class constraints and hence gauge symmetries per spacetime point has been reduced by one.

The first, most naive temptation how to eliminate this discrepancy and get closer to general relativity is to restore the full dependence of the lapse function on space and time by 
hand. This option, often referred to in the literature as the "nonprojectable case" [1,2], can be viewed at least from two different perspectives, which lead to different results.

First, one can follow the logic of effective field theory: Having postulated a multiplet of spacetime fields

$$
g_{i j}(t, \mathbf{x}), \quad N_{i}(t, \mathbf{x}), \quad N(t, \mathbf{x}),
$$

we postulate a list of global and gauge symmetries, and construct the most general action allowed. In the case at hand, the natural gauge symmetries are the foliation-preserving diffeomorphisms $\operatorname{Diff}(M, \mathcal{F})$. While $(1.19)$ is invariant under $\operatorname{Diff}(M, \mathcal{F})$, it is not the most general Hamiltonian compatible with these gauge symmetries. As was pointed out already in $[1,2]$ and further elaborated in $[8,9]$, in this effective field theory approach to the nonprojectable theory, all terms compatible with the gauge symmetry should be allowed in the Lagrangian. Promoting the lapse function to a spacetime field gives a new ingredient for constructing gauge-invariant terms in the action,

$$
\nabla_{i} N / N
$$

which transforms under $\operatorname{Diff}(M, \mathcal{F})$ as a spatial vector and a time scalar. Once terms with this new ingredient are allowed in the action, the Hamiltonian is no longer linear in $N$, but the algebra of constraints is well-behaved [18]. The constraint implied by the variation of $N$ is now second-class, and the expected number of propagating degrees of freedom is the same as in the minimal theory.

Another possible interpretation of the nonprojectable theory also starts by promoting $N$ to a spacetime-dependent field $N(t, \mathbf{x})$. Instead of specifying a priori gauge symmetries, however, one can postulate that the Hamiltonian take the form (1.19), linear in $N$ [19]. This step must be followed by the analysis of the algebra of Hamiltonian constraints, which determines a posteriori whether this construction is consistent, and if so, what is the resulting structure of the gauge symmetries. Here the difficulty is in closing the constraint algebra [1,19-21] (see also [22]): For general $\mathcal{V}$, the commutator of $\mathcal{H}_{0}(\mathbf{x})$ with $\mathcal{H}_{0}(\mathbf{y})$ is a complicated function of all variables, and the requirement of closure is difficult to implement. One interesting exception has been found in the infrared limit [23] (see also [19,24]): Adding $\pi \equiv g_{i j} \pi^{i j}$ as another constraint closes the algebra, turning $\pi$ and $\mathcal{H}_{0}$ into a pair of second-class constraints. This infrared theory can then be interpreted as general relativity whose gauge freedom has been partially fixed. This is a very appealing picture, but the problem is that it cannot be straightforwardly extended to the full theory beyond the infrared limit. However, as we will see below, the possibility of interpreting $\pi$ as an additional constraint in the infrared regime as suggested in [23] will be echoed in the generally covariant theory which we present in Section 1 .

In addition to the two perspectives just reviewed, there is another option how to close the constraint algebra in the nonprojectable theory, and interpret it as a topological field theory. ${ }^{6}$ This is possible when the theory satisfies the detailed balance condition $[1,2]$, i.e., when the

\footnotetext{
${ }^{6}$ This option was pointed out by one of us in [20]; see also Section 5.4 of [1].
} 
potential $\mathcal{V}$ in $(1.15)$ is of the special form

$$
\mathcal{V}=\frac{1}{4} \mathcal{G}_{i j k \ell} \frac{\delta W}{\delta g_{i j}} \frac{\delta W}{\delta g_{k \ell}}
$$

for some action functional $W\left(g_{i j}\right)$ which depends only on $g_{i j}$ and its spatial derivatives. In such cases, it is convenient to introduce a system of complex variables, defined as

$$
a^{i j}=\mathrm{i} \pi^{i j}+\frac{1}{\kappa^{2}} \frac{\delta W}{\delta g_{i j}}, \quad \bar{a}^{i j}=-\mathrm{i} \pi^{i j}+\frac{1}{\kappa^{2}} \frac{\delta W}{\delta g_{i j}} .
$$

Under the Poisson bracket, these variables play essentially the role of a creation and annihilation pair, their only nonzero bracket being

$$
\left[a^{i j}(\mathbf{x}), \bar{a}^{k \ell}(\mathbf{y})\right]=-\frac{2 \mathrm{i}}{\kappa^{2}} \frac{\delta^{2} W}{\delta g_{i j}(\mathbf{x}) \delta g_{k \ell}(\mathbf{y})} .
$$

The Hamiltonian constraints $\mathcal{H}_{i}$ and $\mathcal{H}_{0}$ can be expressed as simple functions of the complex variables,

$$
\mathcal{H}^{i}=\mathrm{i} \nabla_{j}\left(a^{i j}-\bar{a}^{i j}\right), \quad \mathcal{H}_{0}=\frac{\kappa^{2}}{2} a^{i j} \mathcal{G}_{i j k \ell} \bar{a}^{k \ell} .
$$

The problematic commutator of $\mathcal{H}_{0}(\mathbf{x})$ and $\mathcal{H}_{0}(\mathbf{y})$ is still rather complicated, but it clearly vanishes when $a^{i j}$ or $\bar{a}^{i j}$ vanish. The constraint algebra can thus be closed by declaring $\mathcal{H}_{i}$, together with either $a^{i j}$ or $\bar{a}^{i j}$ to be the primary constraints. This would then guarantee that the original Hamiltonian constraints $\mathcal{H}_{i}$ and $\mathcal{H}_{0}$, as well as all their commutators, are zero on the constraint surface.

This step can be made more precise as follows. Because $a^{i j}$ and $\bar{a}^{i j}$ are complex conjugates of each other, it is not possible to declare only (say) $a^{i j}$ to be first-class constraints, at least not without making $a^{i j}$ and $\bar{a}^{i j}$ formally independent. Instead, we accomplish our goal by declaring both $a^{i j}$ and $\bar{a}^{i j}$ as constraints. Because their commutator (1.24) is nonzero, these constraints are second-class and do not imply any additional gauge symmetry.

However, a pair of second-class constraints can often be interpreted as a first-class constraint, together with a gauge-fixing condition. We can interpret the theory with the secondclass constraints $a^{i j}$ and $\bar{a}^{i j}$ in this fashion: First, we choose $a^{i j}-\bar{a}^{i j}$ as the first-class constraint. The gauge symmetry generated by this constraint acts on $g_{i j}$ via

$$
\delta g_{i j}=\lambda_{i j}(t, \mathbf{x})
$$

with $\lambda_{i j}$ an arbitrary spacetime-dependent symmetric two-tensor. This is just the topological gauge symmetry as introduced originally by Witten $[25,26]$, here acting on the spatial component of the metric. The theory is then fully specified by the choice of a gauge-fixing condition for the topological gauge symmetry. In our case, this choice should restore $a^{i j}$ and $\bar{a}^{i j}$ as second-class constraints. Choosing $a^{i j}+\bar{a}^{i j}$ as the gauge fixing condition is certainly a consistent possibility; however, a more interesting scenario is available when we Wick-rotate the theory to imaginary time, $t=-i \tau$. This case is of particular interest because topological 
field theories are typically formulated in imaginary time. In this regime, $a^{i j}$ and $\bar{a}^{i j}$ are now real, instead of being complex conjugates. We can then select an asymmetric gauge-fixing condition, for example

$$
a^{i j} \equiv-\pi^{i j}+\frac{1}{\kappa^{2}} \frac{\delta W}{\delta g_{i j}}=0 .
$$

This equation is a flow equation for $g_{i j}$ as a function of the imaginary time $\tau$, reminiscent of the Ricci flow equation and its cousins. We have thus obtained a topological field theory associated with the flow equations on Riemannian manifolds. Indeed, the number of topological gauge symmetries (1.26) is the same as the number of field components of $g_{i j}$ : The theory has no local propagating degrees of freedom in the bulk.

The original $\operatorname{Diff}(M, \mathcal{F})$ symmetry can be viewed as a separate gauge symmetry in addition to the topological gauge symmetry (1.26). However, because the action (1.11) of $\operatorname{Diff}(M, \mathcal{F})$ on $g_{i j}$ is a special case of $(1.26)$, including $\operatorname{Diff}(M, \mathcal{F})$ explicitly leads to a redundancy in gauge symmetries, and triggers the appearance of "ghost-for-ghosts" in the BRST formalism. In this respect, the structure of the gauge symmetries is very similar to the conventional topological field theories of the cohomological type [25,26] such as topological Yang-Mills theory.

In this paper, we are interested in gravity with bulk propagating degrees of freedom, whose spectrum contains the tensor polarizations of the graviton but not the scalar mode. Therefore, we do not pursue the nonprojectable theory further, and look for the missing gauge invariance elsewhere.

\section{Global $U(1)_{\Sigma}$ Symmetry in the Minimal Theory at $\lambda=1$}

In general relativity, the value $\lambda=1$ of the coupling in (1.15) is selected - and protected from renormalization - by the gauge symmetries $\operatorname{Diff}(M)$ of the theory. It is perhaps surprising that the case of $\lambda=1$ plays a special role in the minimal theory with anisotropic scaling as well $[1,2]$. One can see this by examining the spectrum of the linarized fluctuations around the flat space solution.

For simplicity, we will now assume that the flat spacetime

$$
g_{i j}=\delta_{i j}, \quad N_{i}=0, \quad N=1
$$

is a solution of the equations of motion, and expand the metric to linear order around this background,

$$
g_{i j}=\delta_{i j}+\kappa h_{i j}, \quad N_{i}=\kappa n_{i}, \quad N=1+\kappa n .
$$

Since $n$ is not a spacetime field but only a function of time, its equation of motion gives one integral constraint, and does not affect the number of local degrees of freedom or their dispersion relations. Therefore, we only consider the equations of motion for the spacetime fields $h_{i j}$ and $n_{i}$. 
It will be convenient to further decompose the $h_{i j}$ and $n_{i}$ fluctuations into their irreducible components,

$$
h_{i j}=s_{i j}+\partial_{i} w_{j}+\partial_{j} w_{i}+\left(\partial_{i} \partial_{j}-\frac{1}{D} \delta_{i j} \partial^{2}\right) B+\frac{1}{D} \delta_{i j} h
$$

where the scalar $h=h_{i i}$ is the trace part of $h_{i j}$, while $s_{i j}$ is symmetric, traceless and transverse (i.e., divergence-free: $\partial_{i} s_{i j}=0$ ), and $w_{i}$ is transverse; and similarly,

$$
n_{i}=u_{i}+\partial_{i} C
$$

with $u_{i}$ transverse, $\partial_{i} u_{i}=0$. It is also useful to decompose the linearized gauge transformations,

$$
\xi^{i}(\mathbf{x}, t)=\zeta_{i}(\mathbf{x}, t)+\partial_{i} \eta(\mathbf{x}, t) .
$$

In this decomposition, $\zeta^{i}$ satisfy $\partial_{i} \zeta_{i}=0$, and therefore represent the generators of linearized volume-preserving spatial diffeomorphisms. The linearized gauge transformations act on the irreducible components of the fields via

$$
\begin{aligned}
& \delta s_{i j}=0, \quad \delta w_{i}=\zeta_{i}, \quad \delta B=2 \eta, \quad \delta h=2 \partial^{2} \eta, \\
& \delta u_{i}=\dot{\zeta}_{i}, \quad \delta C=\dot{\eta} .
\end{aligned}
$$

These rules suggest a few natural gauge-fixing conditions. For example, we can set $u_{i}=0$ and $C=0$, which leaves the residual symmetries with time-independent $\zeta_{i}(\mathbf{x})$ and $\eta(\mathbf{x})$, or $w_{i}=0$ and $B=0$, which fixes the gauge symmetries completely. In either gauge, the spectrum of linearized fluctuations around the flat background contains transverse traceless polarizations $s_{i j}$ which all share the same dispersion relation (dependent on the details of $\mathcal{V}$ ), and a scalar whose dispersion is dependent on $\lambda$. In the vicinity of $\lambda=1$, the dispersion relation of the scalar graviton exhibits a singular behavior,

$$
\omega^{2}=(\lambda-1) F\left(\mathbf{k}^{2}, \lambda\right),
$$

where $F\left(\mathbf{k}^{2}, \lambda\right)$ is a regular function of $\lambda$ near $\lambda=1$, whose details again depend on $\mathcal{V}$. Thus, the scalar dispersion relation degenerates to $\omega^{2}=0$ in the limit of $\lambda \rightarrow 1[1,2]$.

\subsection{Symmetries in the linearized approximation around flat spacetime}

The spectrum of linear excitations around the flat spacetime shows that the relativistic value $\lambda=1$ is special even in the nonrelativistic theory, as indicated by the dispersion relation of the scalar graviton mode (2.7) which degenerates as $\lambda \rightarrow 1$. This singular behavior was explained in [1]: At $\lambda=1$, the linearized theory with $\lambda=1$ enjoys an interesting Abelian symmetry, which acts on the fields of the minimal theory via

$$
\delta n_{i}=\partial_{i} \alpha, \quad \delta h_{i j}=0, \quad \delta n=0 .
$$

Here the parameter $\alpha(\mathbf{x})$ is an arbitrary smooth function of the spatial coordinates, constant in time:

$$
\dot{\alpha}=0
$$


Since the generator $\alpha$ is independent of time, it is natural to interpret this infinite-dimensional Abelian symmetry as a global symmetry: In the nonrelativistic setting, it is the hallmark of gauge symmetries in the Lagrangian formalism that their generators are arbitrary functions of time. In order to indicate that the Abelian symmetries generated by $\alpha(\mathbf{x})$ represent a collection of $U(1)$ symmetries parametrized by the spatial slice $\Sigma$ of the spacetime foliation, we will refer to this infinite-dimensional symmetry by $U(1)_{\Sigma}$.

At this stage, it is interesting to note that the $U(1)_{\Sigma}$ symmetry looks very reminiscent of a residual gauge symmetry in a gauge theory, in which some sort of temporal gauge has been chosen. As we will see in the rest of the paper, this intuition is essentially correct, but the specific realization of this idea in the full nonlinear theory will be surprisingly subtle.

In order to see that $U(1)_{\Sigma}$ is indeed a symmetry of the linearized theory at $\lambda=1$, it is instructive to restore temporarily $\lambda$, and evaluate the variation of the action under (2.8) in the linearized approximation (which we denote by “ $\approx$ "), while allowing $\alpha$ to be time dependent:

$$
\delta_{\alpha} S \approx 2 \int d t d^{D} \mathbf{x}\left\{\dot{\alpha}\left(\frac{D-1}{D}\left(\partial^{2}\right)^{2} B+\frac{1-\lambda D}{D} \partial^{2} h\right)-2 \alpha(\lambda-1)\left(\partial^{2}\right)^{2} C\right\} .
$$

At $\lambda=1$, the last term drops out, and the action is invariant under time-independent $\alpha$. Note also that the term proportional to $\dot{\alpha}$ in (2.10) is not gauge invariant under (2.6), unless $\lambda=1$ when it equals

$$
\frac{D-1}{D}\left\{\left(\partial^{2}\right)^{2} B-\partial^{2} h\right\} \approx R,
$$

which we recognize as the linearized Ricci scalar of $g_{i j}$.

Given this global $U(1)_{\Sigma}$ symmetry, it is natural to ask whether it can be gauged. At $\lambda=1$, this process can be easily completed in the linearized theory. We promote $\alpha$ to an arbitrary smooth function of $\mathbf{x}$ and $t$, introduce a gauge field $A(\mathbf{x}, t)$, and postulate its transformation rules under the gauge transformations,

$$
\delta_{\alpha} A=\dot{\alpha}
$$

The gauging is accomplished by augmenting the action by a coupling of $A$ to the linearized Ricci scalar,

$$
S_{A}=-\frac{2(D-1)}{D} \int d t d^{D} \mathbf{x} A\left\{\left(\partial^{2}\right)^{2} B-\partial^{2} h\right\} .
$$

It is easy to see that in the gauged theory, the scalar mode of the graviton has been eliminated from the spectrum of physical excitations: With $A=0$ as our gauge choice, the equations of motion are the same as in the original theory with the global $U(1)_{\Sigma}$ symmetry, plus the Gauss constraint

$$
\left(\partial^{2}\right)^{2} B-\partial^{2} h=0 .
$$

This Gauss constraint eliminates the scalar degree of freedom, leaving only the tensor modes of the graviton in the physical spectrum of the linearized theory. 


\subsection{The nonlinear theory}

We would now like to extend the success of the $U(1)$ gauging from the linearized approximation to the full nonlinear theory. Before we can proceed with the gauging, however, we must first check whether $U(1)_{\Sigma}$ extends to a global symmetry of the nonlinear theory.

In the linearized theory before gauging, the parameter $\alpha(\mathbf{x})$ of the infinitesimal $U(1)_{\Sigma}$ transformation was independent of time, and consequently we interpreted $U(1)_{\Sigma}$ as a global symmetry. In the nonlinear theory, the linearized transformation of $n_{i}$ in (2.8) simply becomes $^{7}$

$$
\delta_{\alpha} N_{i}=N \nabla_{i} \alpha
$$

However, the condition (2.9) expressing the time independence of $\alpha$ is not covariant under $\operatorname{Diff}(M, \mathcal{F})$. The correct covariant generalization takes the following modified form,

$$
\dot{\alpha}-N^{i} \nabla_{i} \alpha=0 .
$$

This condition of vanishing covariant time derivative of $\alpha$ is indeed invariant under $\operatorname{Diff}(M, \mathcal{F})$.

In the full nonlinear theory, the gauge field will transform as a spatial scalar and a time vector under $\operatorname{Diff}(M, \mathcal{F})$,

$$
\delta A=\dot{f} A+f \dot{A}+\xi^{i} \partial_{i} A,
$$

and the gauge transformation of the gauge field becomes

$$
\delta_{\alpha} A=\dot{\alpha}-N^{i} \nabla_{i} \alpha
$$

It follows from (2.18) and (1.16) that the scaling dimensions of $\alpha$ and $A$ are given by

$$
[\alpha]=z-2, \quad[A]=2 z-2 .
$$

In the process of evaluating the variation of the action under the general $\alpha$ transformation, we will encounter a particular combination of the second spatial derivatives of $\dot{g}_{i j}$, which can be expressed as the trace of the time derivative of the Ricci tensor:

$$
g^{i j} \dot{R}_{i j}=\left(g^{i k} g^{j \ell}-g^{i j} g^{k \ell}\right) \nabla_{i} \nabla_{j} \dot{g}_{k \ell}
$$

This formula also implies

$$
(\sqrt{g} R)^{\cdot}=-\sqrt{g}\left(R^{i j}-\frac{1}{2} R g^{i j}\right) \dot{g}_{i j}+\sqrt{g}\left(g^{i k} g^{j \ell}-g^{i j} g^{k \ell}\right) \nabla_{i} \nabla_{j} \dot{g}_{k \ell} .
$$

\footnotetext{
${ }^{7}$ The explicit multiplicative factor of $N$ in 2.15 is explained by the requirement of matching the tensorial properties of both sides in (2.15) under $\operatorname{Diff}(M, \mathcal{F})$. Thus, it is in fact $A_{i} \equiv N_{i} / N$ that transforms as the spatial projection of a spacetime vector field under $\operatorname{Diff}(M, \mathcal{F})$ and as the spatial part of a gauge potential under $U(1)$, with $\delta A_{i}=\nabla_{i} \alpha$.
} 
It is now straightforward to see that there is an obstruction against extending the global symmetry to the full nonlinear theory, at least in dimensions greater than $2+1$. Indeed, for the variation of the action we get

$$
\begin{gathered}
\delta_{\alpha} S=-\frac{1}{\kappa^{2}} \int d t d^{D} \mathbf{x} \sqrt{g}\left(\dot{g}_{i j}-\nabla_{i} N_{j}-\nabla_{j} N_{i}\right)\left(g^{i k} g^{j \ell}-g^{i j} g^{k \ell}\right)\left(\nabla_{k} \nabla_{\ell} \alpha+\nabla_{\ell} \nabla_{k} \alpha\right) \\
=-\frac{2}{\kappa^{2}} \int d t d^{D} \mathbf{x} \sqrt{g} \alpha\left(g^{i j} \dot{R}_{i j}-2 G^{i j k \ell} \nabla_{k} \nabla_{\ell} \nabla_{i} N_{j}\right)
\end{gathered}
$$

where in the second line we have integrated by parts twice, dropped the corresponding spatial derivative terms, and used (2.20). The last, triple-derivative term in (2.22) can be simplified using

$$
G^{i j k \ell} \nabla_{k} \nabla_{\ell} \nabla_{i} N_{j}=-\nabla^{j}\left[\nabla_{j}, \nabla_{k}\right] N^{k}+\frac{1}{2}\left[\nabla_{k}, \nabla_{j}\right] \nabla^{j} N^{k}=\nabla_{j}\left(R^{j k} N_{k}\right),
$$

which yields

$$
\delta_{\alpha} S=-\frac{2}{\kappa^{2}} \int d t d^{D} \mathbf{x} \sqrt{g} \alpha\left\{g^{i j} \dot{R}_{i j}-2 \nabla_{j}\left(R^{j k} N_{k}\right)\right\} .
$$

Finally, after using the contracted Bianchi identity in the second term, integrating by parts in both terms, using (2.21) and dropping the total derivatives, we obtain

$$
\begin{aligned}
\delta_{\alpha} S=\frac{2}{\kappa^{2}} \int d t & d^{D} \mathbf{x} \sqrt{g}\left(\dot{\alpha}-N^{i} \nabla_{i} \alpha\right) R \\
& -\frac{2}{\kappa^{2}} \int d t d^{D} \mathbf{x} \sqrt{g} \alpha\left(R^{i j}-\frac{1}{2} R g^{i j}\right)\left(\dot{g}_{i j}-\nabla_{i} N_{j}-\nabla_{j} N_{i}\right) .
\end{aligned}
$$

The first line in (2.24) vanishes for the covariantly time-independent $\alpha$ by virtue of (2.16), but the second line represents an obstruction against the invariance of $S$, even when $\alpha$ is restricted to be covariantly time-independent.

Another way of seeing the origin of the nonlinear obstruction against the $U(1)_{\Sigma}$ invariance of the minimal theory is the following. On the components $N_{i}$ of the shift vector, the $U(1)_{\Sigma}$ transformations act as gauge transformations on the components of an Abelian connection. Define

$$
F_{i j}=\partial_{i} N_{j}-\partial_{j} N_{i}
$$

Clearly, this is the field strength of $N_{i}$ interpreted as a connection associated with $U(1)_{\Sigma}$. Thus, $F_{i j}$ are invariant under $U(1)_{\Sigma}$, and transform as components of a two-form under $\operatorname{Diff}(\Sigma)$. However, $F_{i j}$ do not transform as two-form components under time-dependent spatial diffeomorphisms.

In this new notation, the action with $\lambda=1$ can be rewritten as

$$
\begin{aligned}
S=\frac{1}{2 \kappa^{2}} \int d t d^{D} \mathbf{x} \frac{\sqrt{g}}{N}\left\{\dot{g}_{i j}\left(g^{i k} g^{j \ell}-g^{i j} g^{k \ell}\right) \dot{g}_{k \ell}-F^{i j} F_{i j}\right. \\
\left.-4 R^{i j} N_{i} N_{j}+4 N^{i}\left(\nabla^{j} \dot{g}_{i j}-g^{j k} \nabla_{i} \dot{g}_{j k}\right)\right\}-\frac{2}{\kappa^{2}} \int d t d^{D} \mathbf{x} \sqrt{g} N \mathcal{V}
\end{aligned}
$$


While the first two terms in (2.26) and the potential term are manifestly invariant under $U(1)_{\Sigma}$, the terms with explicit factors of $N_{i}$ - which are required by the requirement of $\operatorname{Diff}(M, \mathcal{F})$ invariance - are not, and their variation reproduces (2.24). Intuitively, the obstruction can be related to the fact that $N_{i}$ plays a dual role in the theory. First, as we have seen in (1.12), $N_{i}$ is the gauge field of the time-dependent spatial diffeomorphisms along $\Sigma$. The second role is asked of $N_{i} / N$ in our attempt to extend the gauge symmetry, and make $N_{i} / N$ transform as the spatial components of a $U(1)$ gauge field.

\section{Gauging the $U(1)_{\Sigma}$ Symmetry: First Examples}

Our intention is to gauge the action of the global $U(1)_{\Sigma}$ in the minimal theory with $\lambda=1$. As we have seen in Section 4.1.3, such gauging is possible in the linearized approximation, and it has the desired effect of eliminating the scalar polarization of the graviton. However, in Section 2.2 we found an obstruction which prevents $U(1)_{\Sigma}$ from being a global symmetry of the minimal theory at the nonlinear level, and therefore precludes its straightforward gauging. More precisely, we found that the variation (2.24) of the action under an infinitesimal $U(1)$ gauge transformation $\alpha(t, \mathbf{x})$ consists of two parts,

$$
\frac{2}{\kappa^{2}} \int d t d^{D} \mathbf{x} \sqrt{g}\left(\dot{\alpha}-N^{i} \nabla_{i} \alpha\right) R
$$

and

$$
-\frac{2}{\kappa^{2}} \int d t d^{D} \mathbf{x} \sqrt{g} \alpha\left(R^{i j}-\frac{1}{2} R g^{i j}\right)\left(\dot{g}_{i j}-\nabla_{i} N_{j}-\nabla_{j} N_{i}\right) .
$$

If $U(1)_{\Sigma}$ were a global symmetry, the first step of the Noether procedure would be to add a Noether coupling term to the action,

$$
S_{A}=-\frac{2}{\kappa^{2}} \int d t d^{D} \mathbf{x} \sqrt{g} A R
$$

The $U(1)$ variation (2.18) of $A$ in (3.3) indeed cancels (3.1). However, at this stage the Noether procedure breaks down, and (3.2) represents the obstruction against gauge invariance.

In order to allow a straightforward gauging on $U(1)_{\Sigma}$, we will have to find a mechanism which eliminates this obstruction. Before proceeding with that, we first examine the geometric origin of $U(1)_{\Sigma}$ as a natural gauge symmetry, and consider several illustrative cases in which the gauging can be completed because the obstruction automatically vanishes. These include a generally covariant nonrelativistic gravity theory in $2+1$ dimensions, and an interacting Abelian theory of gravity in general dimensions.

\subsection{Geometric interpretation of the $U(1)$ symmetry}

The transformation rules (1.11) of $\operatorname{Diff}(M, \mathcal{F})$ on the gravity fields can be systematically derived $[1,2]$ from the action of relativistic diffeomorphisms $\operatorname{Diff}(M)$ on the spacetime metric $g_{\mu \nu}$, as the leading order in the nonrelativistic $1 / c$ expansion. 
It was already noted in [2] that the gauge field $A(t, \mathbf{x})$ and the $U(1)$ symmetry - which we introduced in a rather ad hoc fashion in Sections 2 and 3 - both acquire a natural geometric interpretation in the framework of the $1 / c$ expansion: It turns out that $A$ is simply the subleading term in the $1 / c$ expansion of the relativistic lapse function, and $U(1)$ corresponds to the subleading, linearized spacetime-dependent time reparametrization symmetry of the relativistic theory. In this section, now make these observations more precise.

In Section 1.3, we reviewed some of the difficulties faced in the attempts to promote the lapse function to a spacetime field,

$$
N(t) \rightarrow N(t, \mathbf{x})
$$

In physical terms, the attempts to restore $N$ as a spacetime field can be motivated by the desire to restore the information carried by the Newton potential in general relativity (for generic gauge choices). The geometric understanding of the gauge field $A$ and the gauge symmetry $U(1)$ shows how the generally covariant theory with $U(1) \ltimes \operatorname{Diff}(M, \mathcal{F})$ symmetry restores the Newton potential and avoid the difficulties of the nonprojectable theory: Instead of promoting $N(t)$ into a spacetime field as in (3.4), we keep $N(t)$ as the leading term of the lapse, and introduce the subleading term $A(t, \mathbf{x})$ in the $1 / c$ expansion, at the order in which the Newton potential enters in the nonrelativistic approximation to general relativity:

$$
N(t) \rightarrow N(t)-\frac{1}{c^{2}} A(t, \mathbf{x})
$$

Thus, it is only the subleading part of lapse that becomes a spacetime field.

It is useful to stress that the $1 / c$ formalism of this section is just a trick, whose sole purpose is to provide a geometric explanation of the action of $U(1) \ltimes \operatorname{Diff}(M, \mathcal{F})$, by taking the formal $c \rightarrow \infty$ limit of the relativistic $\operatorname{Diff}(M)$ symmetry. The "speed of light" $c$ is a formal expansion parameter, and should not be confused with the physical speed of light which will be generated in our theory at large distances as a result of the relevant deformations.

\subsubsection{The gauge field $A$ and the Newton potential}

In order to reproduce the field content of the theory, and the transformation rules under the gauge symmetries, we start with a relativistic spacetime metric, and expand it in the powers of $1 / c$ as follows:

$$
g_{\mu \nu}=\left(\begin{array}{cc}
-N^{2}+\frac{N_{i} N^{i}}{c^{2}}+\frac{2 N A}{c^{2}}+\ldots, & \frac{N_{i}}{c}+\ldots \\
\frac{N_{i}}{c}+\ldots, & g_{i j}+\ldots
\end{array}\right)
$$

This step is complemented by a similar expansion of the relativistic spacetime diffeomorphisms with generators $\zeta^{\mu}$,

$$
\zeta^{0}=c f(\mathbf{x}, t)-\frac{1}{c} \frac{\alpha(\mathbf{x}, t)}{N}+\ldots, \quad \zeta^{i}=\xi^{i}(\mathbf{x}, t)+\ldots
$$


In both cases, “..." refer to terms suppressed by $1 / c^{2}$ compared to those displayed. In the transformation rules, the derivative with respect to the relativistic time coordinate is written as $\partial / \partial x^{0}=(1 / c) \partial / \partial t$; it is then the nonrelativistic time $t$ which is held fixed as $c \rightarrow \infty$.

Taking the $c \rightarrow \infty$ limit first requires $\partial_{i} f=0$, which means that the infinitesimal time reparametrizations $f(t)$ are restricted to be only functions of time. In addition, in accord with (3.5), we insist that $N$ be only a function of time. The transformation rules (1.11) under the foliation-preserving diffeomorphisms then follow from the $c \rightarrow \infty$ limit of the spacetime diffeomorphism symmetry. In addition, we get

$$
\begin{aligned}
\delta_{\alpha}\left(\frac{N_{i}}{N}\right) & =\nabla_{i} \alpha \\
\delta_{\alpha} A & =\dot{\alpha}-N^{i} \nabla_{i} \alpha,
\end{aligned}
$$

with all other fields invariant under $\delta_{\alpha}$. We see that the $U(1)$ gauge symmetry of interest is geometrically interpreted as the subleading part of time reparametrizations in the nonrelativistic limit of spacetime diffeomorphisms in general relativity.

This embedding of the gauge field $A$ into the geometric framework of the $1 / c$ expansion sheds additional light on the physical role of $A$ in the theory. Recall that in the leading order of the Newtonian approximation to general relativity, the $g_{00}$ component of the spacetime metric (in the natural gauge adapted to this approximation) is related to the Newton potential $\Phi$ via

$$
g_{00}=-\left(1+\frac{1}{c^{2}} 2 \Phi+\ldots\right)
$$

Comparing this to (3.6), we find that our gauge field $A$ effectively plays the role of the Newton potential,

$$
A=-\Phi+\ldots
$$

As we will see in Section (5.1), this relationship is corrected by higher order terms already at the next order in the post-Newtonian approximation.

\subsubsection{Extending the $1 / c$ expansion}

We can also keep the subleading terms in the spatial metric, replacing

$$
g_{i j} \rightarrow g_{i j}-\frac{1}{c^{2}} \frac{A_{i j}(\mathbf{x}, t)}{N}+\ldots
$$

in (3.6). Following the rules of transformation for the spatial metric to one higher order in $1 / c^{2}$ than before, it turns out that $A_{i j}$ also transforms under $\alpha$,

$$
\delta_{\alpha} A_{i j}=\alpha \dot{g}_{i j}+N_{i} \nabla_{j} \alpha+N_{j} \nabla_{i} \alpha .
$$

This transformation property of $A_{i j}$ is just what is needed to remedy the noninvariance of our action under the local $U(1)$ transformations, by introducing a new coupling

$$
\frac{2}{\kappa^{2}} \int d t d^{D} \mathbf{x} \sqrt{g} A_{i j}\left(R^{i j}-\frac{1}{2} R g^{i j}\right) .
$$


Interestingly, this term is also "accidentally" invariant under another Abelian gauge symmetry, which acts on the fields via

$$
\delta A_{i j}=\nabla_{i} \varepsilon_{j}+\nabla_{j} \varepsilon_{i}
$$

The variation of all the other fields under the $\varepsilon_{i}$ symmetry is zero. The total action is invariant under (3.14): The only term in the action which depends on $A_{i j}$ is (3.13), and its invariance under (3.14) is a consequence of the Bianchi identity.

This new gauge symmetry (3.14) also has a natural geometrical origin: In the process of decomposing the relativistic symmetries in the powers of $1 / c$, we could have also kept the subleading terms in spatial diffeomorphisms,

$$
\xi^{i}=\zeta^{i}-\frac{1}{c^{2}} \frac{\varepsilon^{i}(t, \mathbf{x})}{N}+\ldots
$$

The $c \rightarrow \infty$ limit of the relativistic diffeomorphisms then implies precisely the transformation rules (3.14).

It thus appears that by extending the gravity multiplet to include both the Newtonpotential $A(t, \mathbf{x})$ and the field $A_{i j}(t, \mathbf{x})$, we succeeded in finding a formulation of gravity in which the $U(1) \ltimes \operatorname{Diff}(M, \mathcal{F})$ symmetry of "nonrelativistic general covariance" is realized in the full nonlinear theory without obstructions. In addition, we have also seen that this extended gravity multiplet has a clear and natural geometric interpretation in the context of the $1 / c$ expansion. These features make this extended theory potentially attractive, but a closer inspection shows that the number of propagating degrees of freedom has been once again reduced to zero - the theory turns out to be effectively topological. Consequently, the spectrum of bulk gravitons in the low-energy limit will not match the prediction of low-energy general relativity.

In order to see this, and to count reliably the number of degrees of freedom, we turn once more to the Hamiltonian analysis [10]. Because the subleading fields $A$ and $A_{i j}$ that we kept in the $1 / c$ expansion appear in the action without time derivatives, they will all lead to constraints in the Hamiltonian formulation of the theory. The full phase space is parametrized by fields $N_{i}, A, A_{i j}, g_{i j}$ and their canonical momenta $P^{i}, P_{A}, P^{i j}$ and $\pi^{i j}$, implying that

$$
\operatorname{dim} \mathcal{P}=2(D+1)^{2}
$$

per spatial point. 8 The vanishing of the momenta conjugate to $A, A_{i j}$ and $N_{i}$ represents $(D+2)(D+1) / 2$ primary constraints. The condition that the primary constraints be preserved in time yields secondary constraints: Insisting on $\dot{P}_{A}=0$ requires the vanishing of $R$, and similarly $\dot{P}^{i j}=0$ requires the vanishing of $R^{i j}-\frac{1}{2} R g^{i j}$. In addition, as in general relativity, $\dot{P}^{i}=0$ requires $\mathcal{H}_{i}=0$.

Naively, there are thus $D(D+3) / 2+1$ secondary constraints $\mathcal{H}_{i}, R$ and $R^{i j}-\frac{1}{2} R g^{i j}$. However, these are not all independent: $R^{i j}-\frac{1}{2} R g^{i j}$ satisfies the Bianchi identity, and $R$ is

\footnotetext{
${ }^{8}$ There is also the canonical pair consisting of $N(t)$ and its conjugate momentum $P_{0}(t)$, which only yields an integral constraint and can be dropped for the purpose of counting the local degrees of freedom.
} 
proportional to the trace of $R^{i j}-\frac{1}{2} R g^{i j}$, leaving $D(D+1) / 2$ independent secondary constraints.

All the primary and secondary constraints are first-class: Their commutators vanish on the constraint surface. As a result, we have the total number

$$
\mathcal{C}_{1}=(D+2)(D+1) / 2+D(D+1) / 2=(D+1)^{2}
$$

of first-class constraints. This implies, together with (3.16) and invoking (1.1), that the total number of local propagating degrees of freedom is

$$
\mathcal{N}=\frac{1}{2}\left(\operatorname{dim} \mathcal{P}-2 \mathcal{C}_{1}\right)=0
$$

The theory is effectively topological.

Since our primary inerest in this paper is to find a theory whose spectrum of gravitons matches general relativity at long distances, we will not pursue the extended theory in which the gravity multiplet contains the $A_{i j}$ fields, and set $A_{i j}=0$ from now on.

\subsection{Generally covariant nonrelativistic gravity in $2+1$ dimensions}

In $2+1$ dimensions, the Einstein tensor $R_{i j}-\frac{1}{2} R g_{i j}$ of the spatial metric vanishes identically, which means that (3.2) is zero, and there is no obstruction against gauging the $U(1)_{\Sigma}$ symmetry in the full nonlinear theory. The Noether procedure terminates after one step and leads to the following action,

$$
S=\frac{2}{\kappa^{2}} \int d t d^{2} \mathbf{x} \sqrt{g}\left\{N\left(K_{i j} K^{i j}-\lambda K^{2}-\mathcal{V}\right)-A R\right\}
$$

This action exhibits the $U(1) \ltimes \operatorname{Diff}(M, \mathcal{F})$ gauge symmetry of nonrelativistic general covariance in $2+1$ dimensions, for any choice of $\mathcal{V}$.

The extended gauge symmetry eliminates the scalar degree of freedom of the graviton. To see that, it is convenient to select $A=0$ as the gauge choice. In this gauge, the equations of motion are the same as in the minimal model with $\lambda=1$, with the addition of the Gauss constraint

$$
R=0
$$

It is this additional constraint which eliminates the scalar degree of freedom of the minimal theory. Moreover, since in $2+1$ dimensions the scalar graviton was the only local degree of freedom, the generally covariant theory with the extended $U(1) \ltimes \operatorname{Diff}(M, \mathcal{F})$ symmetry has no local propagating graviton polarizations. In this sense, it is akin to several other, much studied models of gravity in $2+1$ dimensions, such as standard general relativity or chiral gravity $[27,28]$.

Because of the absence of physical fluctuations, the geometry of classical solutions in this theory can be expected to be quite rigid, just as in the case of its relativistic cousins in $2+1$ dimensions. In particular, the Gauss constraint (3.20) forces the two-dimensional spatial 
slices to be flat. It is natural to look for deformations of this theory which would at least replace the Gauss constraint with the more general condition of constant spatial curvature, but there appear to be no consistent deformations that could modify the Gauss constraint to

$$
R=2 \Omega,
$$

with $\Omega$ a new coupling constant. However, once we learn in Section 4.2 how to gauge the $U(1)_{\Sigma}$ symmetry in the general case of $D+1$ dimensions, we will also find a mechanism for turning on this new coupling $\Omega$.

\subsection{Self-interacting Abelian gravity}

Another way to eliminate the obstruction against gauging $U(1)_{\Sigma}$ is to linearize the gauge symmetries of the minimal theory. The fields in the theory with linearized gauge symmetries are $h_{i j}, n_{i}$ and $n$. The gauge transformations $\xi_{i}(t, \mathbf{x})$ and $f(t)$ act via

$$
\begin{aligned}
\delta h_{i j} & =\partial_{i} \xi_{j}+\partial_{j} \xi_{i}, \\
\delta n_{i} & =\dot{\xi}_{i}, \\
\delta n & =\dot{f}
\end{aligned}
$$

and represent the Abelian contraction of $\operatorname{Diff}(M, \mathcal{F})$.

The kinetic term (with $\lambda=1$ ) takes the form

$$
S_{K}=\frac{1}{2} \int d t d^{D} \mathbf{x}\left(\dot{h}_{i j}-\partial_{i} n_{j}-\partial_{j} n_{i}\right)\left(\delta_{i k} \delta_{j \ell}-\delta_{i j} \delta_{k \ell}\right)\left(\dot{h}_{k \ell}-\partial_{k} n_{\ell}-\partial_{\ell} n_{k}\right) .
$$

In this theory, the obstruction (3.2) against gauging vanishes identically, as was already established in our analysis of the linearized approximation to the minimal theory in Section 2.1.

At first glance, it would thus seem that keeping only the linearized gauge symmetries would reduce the model to the nonintreracting Gaussian theory studied in Section 2.1, but in fact it is not so. Even though the kinetic term takes the Gaussian form (3.23), the potential term need not be Gaussian.

Suitable terms in $\mathcal{V}$ are integrals of local operators, which are either invariant under (3.22), or invariant up to a total spatial derivative. The building blocks that can be used to construct such operators are the linearized curvature tensor of the spatial metric, and its derivatives. We will denote the linearized Riemann tensor by

$$
L_{i j k \ell}=\frac{1}{2}\left(\partial_{j} \partial_{k} h_{i \ell}-\partial_{j} \partial_{\ell} h_{i k}-\partial_{i} \partial_{k} h_{j \ell}+\partial_{i} \partial_{\ell} h_{j k}\right),
$$

and similarly the linearized Ricci tensor by $L_{i j} \equiv L_{i k j k}$ and the Ricci scalar by $L \equiv L_{i i}$. Clearly, there is an infinite hierarchy of sutable operators, which reduces to a finite number if we limit the number of spatial derivatives by $2 z$. For interesting values of $z>1$, the general $\mathcal{V}$ built from such terms will not be purely Gaussian, leading to a self-interacting theory.

Thus, in the context of gravity with anisotropic scaling, linearizing the gauge symmetries does not necessarily make the theory noninteracting - we find a novel interacting theory of 
Abelian gravity instead. Curiously, a similar phenomenon has been observed in the case of general relativity 25 years ago by Wald [29], where it was shown that by taking the action to contain higher powers of the linearized curvature, one can construct a self-interacting theory of spin-two fields in flat spacetime with linearized spacetime diffeomorphisms as gauge symmetries. In the relativistic case studied in [29], this construction leads inevitably to higher time derivatives in the action, and therefore problems with ghosts in perturbation theory. In contrast, our nonrelativistic models of self-interacting Abelian gravity do not suffer from this problem - their self-interaction results from higher than quadratic terms in $\mathcal{V}$, with the kinetic term taking the Gaussian form (3.23). For suitable choices of the couplings in $\mathcal{V}$, the spectrum is free of both ghosts and tachyons.

For an arbitrary $\mathcal{V}$, the gauging of $U(1)_{\Sigma}$ is now accomplished by adding a new Gaussian term to the action,

$$
-2 \int d t d^{D} \mathbf{x} A L
$$

The theory is now gauge invariant under the linearized action of $U(1)$,

$$
\delta A=\dot{\alpha}, \quad \delta n_{i}=\partial_{i} \alpha .
$$

Arguments identical to those in Section 2.1 show that the theory contains only the tensor graviton modes, eliminating the scalar.

At long distances, the dominant terms in $\mathcal{V}$ are those with the lowest number of spatial derivatives. Since the only suitable operator with just two derivatives is the quadratic part of the spatial Einstein-Hilbert term,

$$
\int d t d^{D} \mathbf{x}\left(h_{i j} L_{i j}-\frac{1}{2} h_{i i} L\right)
$$

the theory becomes automatically Gaussian at long distances, and approaches a free infrared fixed point with $z=1$. This behavior can be avoided if we insist that all operators $\mathcal{V}$ are integrals of gauge-invariant operators: Since (3.27) is only invariant up to a total derivative, it does not belong to this class. In these restricted theories, the infrared behavior will be controlled by Gaussian terms with $z \geq 2$. In fact, such self-interacting Abelian gravity theories, approaching free-field Lifshitz-type fixed points $z \geq 1$, have been encountered in the infrared regime of a family of condensed matter models on the rigid fcc lattice in [30].

While such self-interacting Abelian gravity models might be useful for describing new universality classes of bose liquids in condensed matter theory, they do not appear phenomenologically viable as candidates for describing the gravitational phenomena in the observed universe.

\section{General Covariance at a Lifshitz Point}

So far, we focused on the special cases in which the obstruction to the gauging of $U(1)_{\Sigma}$ is absent. However, none of the resulting theories of gravity with extended gauge symmetry 
discussed in Section 3 appear phenomenologically interesting as models of gravity in $3+1$ dimensions.

Here we change our perspective, and present a robust mechanism which allows $U(1)_{\Sigma}$ to be gauged in the general spacetime dimension $D+1$. This will lead to a theory of gravity with nonrelativistic general covariance which reproduces many properties of general relativity at long distances.

\subsection{Repairing the global $U(1)_{\Sigma}$ symmetry}

In Section 2.2, we found an obstruction that prevents the $U(1)_{\Sigma}$ from being a global symmetry of the full nonlinear theory for $D>2$. Leaving aside the possibility that this obstruction could be cancelled by quantum effects (perhaps by a mechanism similar to $[31,32]$ ), we look for a way to repair the $U(1)_{\Sigma}$ symmetry at the classical level.

\subsubsection{The Newton prepotential}

In order to eliminate the obstruction, we introduce an auxiliary scalar field $\nu$, which transforms under $U(1)_{\Sigma}$ as

$$
\delta_{\alpha} \nu=\alpha
$$

We will refer to this field as the "Newton prepotential." The scaling dimension of $\nu$ is the same as the dimension of $\alpha$,

$$
[\nu]=z-2 .
$$

We can now repair the $U(1)_{\Sigma}$ symmetry by adding a new term to the action,

$$
\begin{gathered}
S_{\nu}=\frac{2}{\kappa^{2}} \int d t d^{D} \mathbf{x} \sqrt{g} \nu\left(R^{i j}-\frac{1}{2} R g^{i j}\right)\left(\dot{g}_{i j}-\nabla_{i} N_{j}-\nabla_{j} N_{i}\right) \\
+\frac{2}{\kappa^{2}} \int d t d^{D} \mathbf{x} \sqrt{g} N \nu\left(R^{i j}-\frac{1}{2} R g^{i j}\right) \nabla_{i} \nabla_{j} \nu .
\end{gathered}
$$

The variation of $\nu$ in the linear term compensates for the noninvariance of the original action of the minimal theory. The term quadratic in $\nu$ is in turn required to cancel the variation of $N_{i}$ in the term linear in $\nu$.

\subsubsection{Relevant deformations}

We can check by linearizing around the flat background that the number of propagating degrees of freedom has not changed by the introduction of the Newton prepotential terms in the action. It is rather unconventional that in the expansion around the flat spacetime, the new field $\nu$ enters the action at the cubic order in small fields, i.e., its presence does not affect the propagator. This issue is eliminated by noticing that a new term, of lower dimension and also invariant under the global $U(1)_{\Sigma}$ symmetry, can be added to the action:

$$
S_{\Omega}=\frac{2 \Omega}{\kappa^{2}} \int d t d^{D} \mathbf{x} \sqrt{g} \nu g^{i j}\left(\dot{g}_{i j}-\nabla_{i} N_{j}-\nabla_{j} N_{i}\right)+\frac{2 \Omega}{\kappa^{2}} \int d t d^{D} \mathbf{x} \sqrt{g} N \nu \Delta \nu .
$$


Here $\Omega$ is a coupling constant of dimension $[\Omega]=2$. With the addition of this relevant term, the Newton prepotential enters the linearized theory, at the quadratic order in fields around the flat spacetime.

The Newton prepotential enters the action with global $U(1)_{\Sigma}$ symmetry quadratically, and can be integrated out by solving its equation of motion,

$$
\Theta^{i j} \nabla_{i} \nabla_{j} \nu+\Theta^{i j} K_{i j}=0
$$

where we have introduced

$$
\Theta^{i j}=R^{i j}-\frac{1}{2} R g^{i j}+\Omega g^{i j} .
$$

Integrating out $\nu$ would result in a nonlocal action, because (4.5) is solved by

$$
\nu_{0}(\mathbf{x}, t)=-\int d^{D} \mathbf{x}^{\prime} \frac{1}{\Theta^{i j} \nabla_{i} \nabla_{j}}\left(\mathbf{x}, \mathbf{x}^{\prime}\right)\left(\Theta^{k \ell} K_{k \ell}\right)\left(\mathbf{x}^{\prime}\right) .
$$

Here we have assumed that the operator $\Theta^{i j} \nabla_{i} \nabla_{j}$ is invertible, and denoted its Green's function by $\left(\Theta^{i j} \nabla_{i} \nabla_{j}\right)^{-1}\left(\mathbf{x}, \mathbf{x}^{\prime}\right)$. We will not try to determine the exact conditions under which this assumption is true. However, for example near the flat spacetime geometry (2.1), we have $\Theta^{i j} \nabla_{i} \nabla_{j}=\Omega \Delta+\mathcal{O}\left(h_{i j}\right)$, where $\Delta=\partial^{2}$ is the flat-space Laplacian. This operator is invertible at least in perturbation theory, as long as we keep $\Omega$ nonzero. ${ }^{9}$

Note that the Green's function is still a local function in $t$. Note also that the expression (4.7) for $\nu_{0}$ has the right form in order for the action to be $U(1)_{\Sigma}$ invariant after $\nu$ has been integrated out. In particular,

$$
\begin{aligned}
& \delta_{\alpha} \nu_{0}(\mathbf{x}, t)=-\frac{1}{2} \int d^{D} \mathbf{x}^{\prime} \frac{1}{\Theta^{i j} \nabla_{i} \nabla_{j}}\left(\mathbf{x}, \mathbf{x}^{\prime}\right) \Theta^{k \ell}\left(-\nabla_{k} \nabla_{\ell} \alpha-\nabla_{\ell} \nabla_{k} \alpha\right)\left(\mathbf{x}^{\prime}\right) \\
&=\int d^{D} \mathbf{x}^{\prime} \frac{1}{\Theta^{i j} \nabla_{i} \nabla_{j}}\left(\mathbf{x}, \mathbf{x}^{\prime}\right)\left(\Theta^{k \ell} \nabla_{k} \nabla_{\ell} \alpha\right)\left(\mathbf{x}^{\prime}\right)=\alpha(\mathbf{x}, t) .
\end{aligned}
$$

The nonlocality of the action obtained by integrating out $\nu$ is relatively mild: In particular, this nonlocality is purely spatial, along the leaves of the spacetime foliation $\mathcal{F}$. Such nonlocalities are quite common in rather conventional condensed matter systems. Nevertheless, in the rest of the paper, we will keep the action manifestly local, by keeping the Newton prepotential $\nu$ as an independent field instead of integrating it out.

\subsubsection{Linearized theory with global $U(1)_{\Sigma}$ around flat spacetime}

First we will check that our repair of the global $U(1)_{\Sigma}$ symmetry has not changed the count of the number of degrees of freedom. Even with the $\Omega$ coupling turned on, the flat spacetime geometry

$$
g_{i j}=\delta_{i j}, \quad N_{i}=0, \quad N=1, \quad \nu=0
$$

\footnotetext{
${ }^{9}$ Another interesting example, which will be important below when we gauge the $U(1)_{\Sigma}$ symmetry, is the case in which $\hat{g}_{i j}$ is the metric of a maximally symmetric space satisfying $\hat{R}=2 \Omega$. In this reference background, we have $\Theta^{i j} \nabla_{i} \nabla_{j}=2 \Omega \hat{\Delta} / D$ (with $\hat{\Delta}$ the Laplace operator of $\hat{g}_{i j}$ ), which is also invertible for $\Omega \neq 0$.
} 
is still a classical solution of the theory (with $\Lambda=0$ ), and we can expand around it.

The $\nu$ equation of motion is

$$
2 \partial^{2}(\nu-C)+\dot{h}=0 .
$$

The momentum constraints give

$$
\partial^{2}\left(\dot{w}_{i}-u_{i}\right)=0
$$

and

$$
2 \Omega \partial^{2} \nu+\frac{D-1}{D}\left\{\left(\partial^{2}\right)^{2} \dot{B}-\partial^{2} \dot{h}\right\}=0 .
$$

Setting $B=0$ and $w_{i}=0$ fixes gauge completely, and implies (with appropriate boundary conditions at infinity) that $u_{i}=0$ and

$$
2 \Omega \nu=\frac{D-1}{D} \dot{h} .
$$

In this gauge, the remaining equations of motion are

$$
-\ddot{s}_{i j}+\frac{D-1}{D} \delta_{i j} \ddot{h}+2\left(\partial_{i} \partial_{j}-\delta_{i j} \partial^{2}\right) \dot{C}-2 \Omega \delta_{i j} \dot{\nu}-\frac{\delta \mathcal{V}_{2}}{\delta g_{i j}}=0,
$$

where $\mathcal{V}_{2}$ denotes the quadratic part of $\mathcal{V}$ in the linearized theory. Using (4.13), we see that the $\dot{\nu}$ term cancels the $\ddot{h}$ term exactly, allowing one to determine $h$ as a function of $\dot{C}$, and substitute back into (4.10). The resulting equation determines the dispersion relation of the scalar polarization of the graviton. For example, when we set the cosmological constant $\Lambda=0$, the potential term will be dominated at long distances by $\mathcal{V}=-R$, and we get

$$
\frac{\delta \mathcal{V}_{2}}{\delta g_{i j}}=-\partial^{2} s_{i j}+\frac{D-2}{D}\left(\partial_{i} \partial_{j}-\delta_{i j} \partial^{2}\right)\left(\partial^{2} B-h\right) .
$$

The metric equation of motion then implies that

$$
h=\frac{2 D}{2-D} \dot{C},
$$

and the $\nu$ equation of motion gives

$$
\frac{D-1}{\Omega} \partial^{2} \ddot{C}+D \ddot{C}+(D-2) \partial^{2} C=0 .
$$

The spectrum thus contains the transverse, traceless polarizations $s_{i j}$ with dispersion

$$
\omega^{2}=\mathbf{k}^{2}
$$

plus a scalar graviton (described in this gauge by $C$ ) which exhibits the dispersion relation implied by (4.17),

$$
\omega^{2}=-\frac{(D-2) \mathbf{k}^{2}}{D\left\{1-\frac{D-1}{D \Omega} \mathbf{k}^{2}\right\}} .
$$


Note that the scalar mode is inevitably tachyonic at low energies. This is implied by the choice of sign in $\mathcal{V}$, determined from the requirement that the tensor polarizations have the correct-sign dispersion relation (4.18). This tachyonic nature of the scalar mode is not a cause for any concern, because the model discussed here represents only an intermediate stage of our construction - our intention is to gauge the $U(1)_{\Sigma}$ symmetry, which will turn the scalar graviton into a gauge artifact.

Note also that taking the regulating dimensionful coupling $\Omega$ to zero reduces the scalar dispersion relation (4.19) correctly to the singular dispersion $\omega^{2}=0$, observed at $\lambda=1$ in the minimal theory in [1] and in (2.7).

\subsection{Gauging the $U(1)_{\Sigma}$ symmetry}

Having repaired the global $U(1)_{\Sigma}$ symmetry, we can now gauge it. The Noether method closes after just one step; adding

$$
S_{A, \Omega}=-\frac{2}{\kappa^{2}} \int d t d^{D} \mathbf{x} \sqrt{g} A(R-2 \Omega)
$$

to the action makes the theory gauge invariant under the $U(1)$ symmetry. This procedure results in the following action of the generally covariant theory of gravity with anisotropic scaling,

$$
S=\frac{2}{\kappa^{2}} \int d t d^{D} \mathbf{x} \sqrt{g}\left\{N\left[K_{i j} K^{i j}-K^{2}-\mathcal{V}+\nu \Theta^{i j}\left(2 K_{i j}+\nabla_{i} \nabla_{j} \nu\right)\right]-A(R-2 \Omega)\right\}
$$

with $\Theta^{i j}$ a short-hand notation for

$$
\Theta^{i j}=R^{i j}-\frac{1}{2} g^{i j} R+\Omega g^{i j}
$$

Note that in the theory with the Newton prepotential $\nu$, the issue about the possibility of adding the spatial cosmological constant $\Omega$, raised in the generally covariant theory in $2+1$ dimensions at the end of Section 3.2, has been resolved by the introduction of the Newton prepotential.

Note also that in addition to the newly introduced gauge field $A$, the theory contains a composite

$$
a=\dot{\nu}-N^{i} \nabla_{i} \nu+\frac{N}{2} \nabla^{i} \nu \nabla_{i} \nu
$$

which also transforms as a gauge field under the $U(1)$ gauge transformations,

$$
\delta_{\alpha} a=\dot{\alpha}-N^{i} \nabla_{i} \alpha
$$

Moreover, both $A$ and the composite gauge field $a$ share the same transformation properties under the rest of the gauge group,

$$
\delta a=\xi^{i} \partial_{i} a+\dot{f} a+f \dot{a} .
$$


As a result, the gauged action stays gauge invariant if we replace

$$
A \rightarrow(1-\gamma) A+\gamma a
$$

with $\gamma$ a real coefficient.

In fact, the composite field $a$ already made its appearance in the theory with the global $U(1)_{\Sigma}$ symmetry presented in Section 4.1: Up to a total derivative, the relevant term (4.4) can be rewritten as

$$
S_{\Omega}=-\frac{4 \Omega}{\kappa^{2}} \int \sqrt{g} a .
$$

Hence, the substitution (4.26) in the $\int d t d^{D} \mathbf{x} \sqrt{g} A$ term in 4.20 ) will just shift the effective value of $\Omega$. Similarly, substituting (4.26) in the $A R$ term in the Noether coupling (4.20) effectively shifts the coefficients in (4.3).

\subsubsection{Hamiltonian formulation}

The structure of gauge symmetries can be verified by analyzing the algebra of Hamiltonian constraints of the theory. In addition, this analysis will allow us to obtain the precise count of the number of propagating degrees of freedom, using formula (1.1). This approach to the count of the degrees of freedom is usually more accurate and more reliable than our previous analysis of the linearized spectrum around a fixed solution, for at least two reasons. First, it is less background-dependent, because it sidesteps the need to linearize the theory around a fixed solution. Secondly, because it is valid for the full nonlinear theory, it excludes the possible artifacts of the linearized approximation.

We will set $\kappa=1$ to eliminate additional clutter, and denote the canonical momenta conjugate to the spatial metric by $\Pi^{i j}$ :

$$
\Pi^{i j}=\frac{\delta S}{\delta \dot{g}_{i j}}=2 \sqrt{g}\left(K^{i j}-g^{i j} K+\Theta^{i j} \nu\right)=\pi^{i j}+2 \sqrt{g} \Theta^{i j} \nu .
$$

The lower-case $\pi^{i j}$ are reserved for the standard expressions for the canonical momenta in general relativity,

$$
\pi^{i j} \equiv 2 \sqrt{g}\left(K^{i j}-g^{i j} K\right)
$$

The remaining canonical momenta

$$
P^{i}(\mathbf{x}, t) \equiv \frac{\delta S}{\delta \dot{N}_{i}}, \quad p_{\nu}(\mathbf{x}, t) \equiv \frac{\delta S}{\delta \dot{\nu}}, \quad P_{A}(\mathbf{x}, t) \equiv \frac{\delta S}{\delta \dot{A}}, \quad P_{0}(t) \equiv \frac{\delta S}{\delta \dot{N}}
$$

all vanish, and represent the primary constraints. The Poisson brackets are

$$
\begin{array}{rlrl}
{\left[g_{i j}(\mathbf{x}, t), \Pi^{k \ell}(\mathbf{y}, t)\right]} & =\frac{1}{2}\left(\delta_{i}^{k} \delta_{j}^{\ell}+\delta_{i}^{\ell} \delta_{j}^{k}\right) \delta(\mathbf{x}-\mathbf{y}), & \\
{\left[N_{i}(\mathbf{x}, t), P^{j}(\mathbf{y}, t)\right]} & =\delta_{i}^{j} \delta(\mathbf{x}-\mathbf{y}), & {\left[N(t), P_{0}(t)\right]} & =1, \\
{\left[A(\mathbf{x}, t), P_{A}(\mathbf{y}, t)\right]} & =\delta(\mathbf{x}-\mathbf{y}), & {\left[\nu(\mathbf{x}, t), p_{\nu}(\mathbf{y}, t)\right]} & =\delta(\mathbf{x}-\mathbf{y}),
\end{array}
$$

and zero otherwise. 
In the canonical variables, the Hamiltonian is given by

$$
\begin{aligned}
H=\int d^{D} \mathbf{x}\{N & {\left[\frac{1}{2 \sqrt{g}}\left(\Pi^{i j}-2 \sqrt{g} \Theta^{i j} \nu\right) \mathcal{G}_{i j k \ell}\left(\Pi^{k \ell}-2 \sqrt{g} \Theta^{k \ell} \nu\right)\right.} \\
& \left.\left.+2 \sqrt{g} \Theta^{i j} \nabla_{i} \nu \nabla_{j} \nu+2 \sqrt{g} \mathcal{V}\right]-2 N_{i} \nabla_{j} \Pi^{i j}+2 \sqrt{g} A(R-2 \Omega)\right\},
\end{aligned}
$$

where

$$
\mathcal{G}_{i j k \ell}=\frac{1}{2}\left(g_{i k} g_{j \ell}+g_{i \ell} g_{j k}\right)-\frac{1}{D-1} g_{i j} g_{k \ell}
$$

is the inverse of the De Witt metric $G^{i j k \ell}$ of $(1.9)$ for $\lambda=1$.

At this stage, the primary constraints are included in the Hamiltonian with the use of Lagrange multipliers $U_{i}(\mathbf{x}, t), \mathcal{U}(\mathbf{x}, t), U_{A}(\mathbf{x}, t)$, and $U_{0}(t)$,

$$
H \rightarrow \hat{H}=H+\int d^{D} \mathbf{x}\left(U_{i} P^{i}+\mathcal{U} p_{\nu}+U_{A} P_{A}\right)+U_{0} P_{0}
$$

The preservation of the primary constraints under the time evolution given by (4.33) requires that the commutators of the primary constraints with $\hat{H}$ vanish, yielding the following set of secondary constraints which are local in space,

$$
\begin{aligned}
\mathcal{H}^{i} & \equiv\left[\hat{H}, P^{i}\right]=-2 \nabla_{j} \Pi^{i j}, \\
\Phi & \equiv\left[\hat{H}, p_{\nu}\right]=-4 \sqrt{g} N \Theta^{i j} \nabla_{i} \nabla_{j} \nu+4 \sqrt{g} N \Theta^{i j} \mathcal{G}_{i j k \ell} \Theta^{k \ell} \nu-2 N \Theta^{i j} \mathcal{G}_{i j k \ell} \Pi^{k \ell}, \\
\Psi & \equiv\left[\hat{H}, P_{A}\right]=2 \sqrt{g}(R-2 \Omega),
\end{aligned}
$$

and one integral constraint

$$
\begin{aligned}
\int d^{D} \mathbf{x} \mathcal{H}_{0} \equiv\left[\hat{H}, P_{0}\right]=\int d^{D} \mathbf{x}\{ & \frac{1}{2 \sqrt{g}}\left(\Pi^{i j}-2 \sqrt{g} \Theta^{i j} \nu\right) \mathcal{G}_{i j k \ell}\left(\Pi^{k \ell}-2 \sqrt{g} \Theta^{k \ell} \nu\right) \\
& \left.+2 \sqrt{g} \Theta^{i j} \nabla_{i} \nu \nabla_{j} \nu+2 \sqrt{g} \mathcal{V}\right\} .
\end{aligned}
$$

This integral constraint will not affect the number of local degrees of freedom. To avoid unnecessary clutter, we concentrate on the analysis of the local constraints, returning to (4.37) only at the end of this section.

Next, we need to ensure that the secondary constraints are preserved in time. The momentum constraints $\mathcal{H}^{i}$ take formally the same form as in general relativity or in the minimal theory of $[1,2]$. They are indeed preserved in time, albeit in a slightly more intricate way than in the minimal theory or in general relativity. In those cases (see the discussion in Section 4.4 of [1]), the commutator of $\mathcal{H}^{i}$ with $H$ only gets a contribution from the $N_{k} \mathcal{H}^{k}$ terms in $H$. The rest of the commutator between the density of the Hamiltonian and $\mathcal{H}^{i}$ adds up to a total derivative, as a consequence of the transformation properties of a scalar density under spatial diffeomorphisms. Here, the argument is more subtle, and the commutator contains additional terms,

$$
\left[\hat{H}, \mathcal{H}^{i}\right]=-\nabla_{k}\left(N^{k} \mathcal{H}_{i}\right)-\left(\nabla_{i} N^{k}\right) \mathcal{H}_{k}-\left(\nabla^{i} \nu\right) \Phi-\left(\nabla^{i} A\right) \Psi .
$$


However, this expression vanishes on the constraint surface, and no tertiary constraints are produced at this stage.

The time preservation of the secondary constraint $\Phi$ requires the vanishing of

$$
[\hat{H}, \Phi] \equiv 4 \sqrt{g} N \Theta^{i j}\left(\nabla_{i} \nabla_{j}-\mathcal{G}_{i j k \ell} \Theta^{k \ell}\right) \mathcal{U}+[H, \Phi]-U_{0} \frac{\Phi}{N}=0 .
$$

Unlike the conditions for the time preservation of the primary constraints or the $\mathcal{H}^{i}$, condition (4.39) depends explicitly on one of the Lagrange multipliers, $\mathcal{U}$. Therefore, setting $[\hat{H}, \Phi]=0$ yields an equation for $\mathcal{U}$, instead of producing an additional, tertiary constraint. Also, because the commutator

$$
\left[p_{\nu}(\mathbf{x}), \Phi(\mathbf{y})\right]=4 \sqrt{g} N \Theta^{i j}\left(\nabla_{i} \nabla_{j}-\mathcal{G}_{i j k \ell} \Theta^{k \ell}\right) \delta(\mathbf{x}-\mathbf{y})
$$

does not vanish on the constraint surface, $p_{\nu}$ and $\Phi$ represent a pair of second-class constraints.

It now remains to check the condition for the preservation of $\Psi$ in time. After a lengthy calculation, we get

$$
[\hat{H}, \Psi]=+N \nabla_{i} \mathcal{H}^{i}-\Phi-\nabla_{i}\left(N^{i} \Psi\right) .
$$

This expression vanishes on the constraint surface. Again, no tertiary constraint is produced, and the process of generating the full list of constraints stops here.

One might be tempted to expect that $\Psi$ is a first-class constraint, but that expectation is false: The commutator of $\Psi(\mathbf{x})$ and $\Phi(\mathbf{y})$ does not vanish on the constraint surface. Consequently, the first-class and second-class constraints are still entangled, and $\Psi(\mathbf{x})$ itself is a mixture of constraints of both classes. In order to disentangle the constraints, we must first evaluate

$$
\begin{aligned}
{[\Psi(\mathbf{x}), \Phi(\mathbf{y})] } & =-4 \frac{\delta\{\sqrt{g}(R-2 \Omega)(\mathbf{x})\}}{\delta g_{i j}(\mathbf{y})}\left(N \mathcal{G}_{i j k \ell} \Theta^{k \ell}\right)(\mathbf{y}) \\
& =4 \sqrt{g} N \Theta^{i j}\left(\mathcal{G}_{i j k \ell} \Theta^{k \ell}-\nabla_{i} \nabla_{j}\right) \delta(\mathbf{x}-\mathbf{y}) .
\end{aligned}
$$

This is equal, up to a sign, to the commutator of $p_{\nu}$ and $\Phi$ which we obtained in (4.40). Hence, it is natural to define

$$
\mathcal{H}_{A}=\Psi+p_{\nu}
$$

$\mathcal{H}_{A}$ commutes both with $\Phi$ and with $p_{\nu}$, and represents a first-class constraint.

Having identified $\mathcal{H}_{A}$ as the final first-class constraint, we can check that it generates the correct $U(1)$ gauge transformations on the fields. In the Hamiltonian formalism, the gauge transformation generated by a first-class constraint on an arbitrary phase-space variable $\phi$ is given by the commutator of $\phi$ with the corresponding constraint [10], for example

$$
\delta_{\alpha} \phi(\mathbf{x}, t)=-\left[\int d^{D} \mathbf{y} \alpha(\mathbf{y}, t) \mathcal{H}_{A}, \phi(\mathbf{x}, t)\right] .
$$

One can indeed use this Hamiltonian formula to check that the gauge symmetries implied by the first-class constraints reproduce those that we found in the Lagrangian formulation above. 
Given our analysis of the constraints, we can now evaluate the number of degrees of freedom. Altogether, the theory has $\operatorname{dim} \mathcal{P}=D^{2}+3 D+4$ canonical variables per spacetime point. These variables are constrained by $\mathcal{C}_{1}=2 D+2$ first-class constraints $\left(P^{i}, P_{A}, \mathcal{H}^{i}\right.$ and $\left.\mathcal{H}_{A}\right)$, and $\mathcal{C}_{2}=2$ second-class constraints $\left(p_{\nu}\right.$ and $\left.\Phi\right)$. The number of degrees of freedom $\mathcal{N}$ per spacetime point is then given by formula (1.1),

$$
\mathcal{N}=\frac{1}{2}\left(\operatorname{dim} \mathcal{P}-2 \mathcal{C}_{1}-\mathcal{C}_{2}\right)=\frac{1}{2}(D+1)(D-2) .
$$

This correctly reproduces the number of tensor (i.e. transverse, traceless) polarizations of the graviton in $D+1$ spacetime dimensions.

Returning to the integral constraint (4.37), we note that its commutation relations with the rest of the constraint algebra can be read off from the commutators of $H$ obtained above. This follows from the fact that, as in general relativity, the Hamiltonian can be written as a sum of constraints,

$$
H=N \int d^{D} \mathbf{x} \mathcal{H}_{\perp}+\int d^{D} \mathbf{x}\left(N^{i} \mathcal{H}_{i}+A \Psi\right)
$$

Actually, the role of the integral constraint (4.37) deserves to be investigated further. It is plausible that in the theory with nonrelativistic general covariance, where the $U(1)$ gauge symmetry mimics the role of relativistic time reparametrizations, one can choose not to impose the integral constraint on physical states. This would be eqiuvalent to the omission of nonrelativistic time reparametrizations $\delta t=f(t)$ from the gauge symmetries, effectively setting $N(t)=1$. If consistent, this construction would lead to a theory of gravity with nonzero energy levels even in spacetimes with compact spatial slices $\Sigma$. In fact, this situation was already encountered on flat noncompact $\Sigma$ in the context of Abelian gravity in [30]. On noncompact $\Sigma$, the possibility of relaxing the integral Hamiltonian constraint will be closely tied to the structure of consistent boundary conditions at infinity in gravity with anisotropic scaling, whose study has been initiated in [33].

\subsubsection{Linearization around detailed balance}

In principle, our result (4.45) for the number of degrees of freedom $\mathcal{N}$ can be checked by linearizing the theory around a chosen solution, and explicitly counting the number of propagating polarizations. However, in order to investigate the spectrum of the linearized theory after gauging, we cannot use the flat spacetime as a reference background, because it no longer solves the equations of motion if $\Omega$ is not zero.

This makes the analysis of the linearized approximation for general values of the couplings algebraically tedious, and we will not present it here in full generality. Instead, we content ourselves with testing (4.45) in the simpler case when the theory satisfies the detailed balance condition. Hence, we assume that the potential takes the special form

$$
\mathcal{V}=\frac{1}{4} \mathcal{G}_{i j k \ell} \frac{\delta W}{\delta g_{i j}} \frac{\delta W}{\delta g_{k \ell}}
$$


and for concreteness we choose

$$
W=\frac{1}{2 \kappa_{W}^{2}} \int d^{D} \mathbf{x} \sqrt{g}\left(R-2 \Lambda_{W}\right)
$$

Before the $U(1)_{\Sigma}$ is gauged, the theory in detailed balance admits a particularly simple static ground-state solution,

$$
g_{i j}=\hat{g}_{i j}(\mathbf{x}), \quad N=1, \quad N_{i}=0, \quad \nu=0,
$$

where $\hat{g}_{i j}$ is the maximally symmetric spatial metric which solves the equations of motion of $W$

$$
R_{i j}-\frac{1}{2} R g_{i j}+\Lambda_{W} g_{i j}=0 .
$$

In order for this background to be a solution of the theory with the extended $U(1) \ltimes \operatorname{Diff}(M, \mathcal{F})$ gauge symmetry, we must set the spatial cosmological constant $\Omega$ equal to

$$
\Omega=\frac{D}{D-2} \Lambda_{W}
$$

For $\Omega>0$, the ground-state geometry is the Einstein static universe, with spatial slices $\Sigma=S^{D}$. Conversely, when $\Omega<0$, the ground state is the hyperbolic version of the Einstein static universe, with noncompact $\Sigma$. Its curvature tensor satisfies $\hat{R}_{i j}=\frac{2 \Omega}{D} \hat{g}_{i j}$ and $\hat{R}=2 \Omega$.

We now determine the spectrum of linearized perturbations around this class of ground state solutions. The analysis closely parallels that of Sections 2.1 and 4.1.3, and we will be brief. We expand the metric, $g_{i j}=\hat{g}_{i j}+\kappa h_{i j}$, and decompose the linearized fluctuations as in (2.3) and (2.4):

$$
\begin{aligned}
h_{i j} & =s_{i j}+\hat{\nabla}_{i} w_{j}+\hat{\nabla}_{j} w_{i}+\left(\hat{\nabla}_{i} \hat{\nabla}_{j}-\frac{1}{D} \hat{g}_{i j} \hat{\Delta}\right) B+\frac{1}{D} h \hat{g}_{i j}, \\
n_{i} & =u_{i}+\hat{\nabla}_{i} C
\end{aligned}
$$

with $\hat{\nabla}_{i}$ the covariant derivative of $\hat{g}_{i j}$. The $\nu$ equation of motion is

$$
\frac{2 \Omega}{D}\left(\hat{\Delta} \nu+\frac{1}{2} \dot{h}-\hat{\Delta} C\right)=0,
$$

and the momentum constraints give

$$
\begin{aligned}
\left(\hat{\Delta}+\frac{2 \Omega}{D}\right)\left(\dot{w}_{i}-u_{i}\right) & =0, \\
\hat{\nabla}_{i}\left(\frac{2 \Omega}{D} \dot{B}+\frac{D-1}{D}(\hat{\Delta} B-\dot{h})+\frac{4 \Omega}{D} \nu\right) & =0 .
\end{aligned}
$$

To fix the $\operatorname{Diff}(M, \mathcal{F})$ symmetries, we set $w_{i}=B=n=0$. In this gauge, the momentum constraints reduce to

$$
(\hat{\Delta}+2 \Omega / D) u_{i}=0, \quad \hat{\nabla}_{i}[4 \Omega \nu-(D-1) \dot{h}]=0,
$$


which implies, with suitable boundary conditions, that $u_{i}$ is not propagating, and that

$$
\Omega \nu=\frac{D-1}{4} \dot{h} .
$$

Plugging this back into 4.53 yields

$$
\frac{D-1}{4 \Omega} \hat{\Delta} \dot{h}+\frac{1}{2} \dot{h}-\hat{\Delta} C=0 .
$$

Finally, there is the constraint $R-2 \Omega=0$, which plays the role of the Gauss constraint in our gauge $A=0$. Its linearization around our detailed balance background

$$
R-\hat{R} \approx-\frac{1}{D}[(D-1) \hat{\Delta} h+2 \Omega h]=0
$$

shows that $h$ is not propagating. Combining (4.58) with 4.57) then implies that $\hat{\Delta} C=0$. Hence, the only propagating modes are the transverse traceless polarizations of the graviton $s_{i j}$. In particular, the scalar graviton has been eliminated, and the number of physical degrees of freedom agrees with the result of our Hamiltonian analysis (4.45).

\section{Conclusions}

In this paper, we have found a formulation of the theory of gravity with anisotropic scaling in which the gauge symmetry of foliation-preserving diffeomorphisms $\operatorname{Diff}(M, \mathcal{F})$ is enhanced to the symmetry of "nonrelativistic general covariance," $U(1) \ltimes \operatorname{Diff}(M, \mathcal{F})$.

The advantage of this construction is that it relies only on the structure of the kinetic term in the action (1.15) (and, in fact, forces it to take the general-relativistic form with $\lambda=1$ ), while the form of the potential term $\mathcal{V}$ is left unconstrained. Therefore, we can consider the scenario proposed originally in $[1,2]$, in which the theory is defined at short distances by a $z>1$ fixed point (with $\mathcal{V}$ dominated by higher-derivative terms), and is then expected to flow under the influence of relevant terms to $z=1$ and isotropic scaling in the infrared. This classical scenario will of course receive quantum corrections, which could drive the theory outside the range of validity of the covariant action (4.21). In the rest of the paper, we limit our attention to the possibility that the long-distance physics is still described by the same action (4.21), with $\mathcal{V}$ dominated by the most relevant terms (1.18).

The first good news is that, as a result of the extended gauge symmetry, the spectrum contains just the transverse-traceless (tensor) modes of the graviton. The scalar graviton mode of the minimal theory has been eliminated. In $3+1$ spacetime dimensions, the elimination of the scalar mode has an interesting consequence in the short-distance regime of the theory. Recall that in the minimal theory with the potential dominated at short distances by the $z=3$ term (1.17), the scalar mode is the sole physical mode that does not get a contribution to its dispersion relation from (1.17), suggesting that terms with $z>3$ would be required to achieve a UV completion [2]. In the theory with the extended gauge symmetry, 
the scalar mode is a gauge artifact, all physical modes aquire a $z=3$ dispersion relation at short distances from (1.17), and no terms with $z>3$ are needed.

The extended gauge symmetry of the theory with nonrelativistic general covariance has even more interesting consequences at long distances, because it improves the chances that the behavior of our theory can resemble general relativity in this observationally relevant regime. We conclude this paper by previewing how our generally covariant theory compares to general relativity at long distances, focusing on the case of $3+1$ spacetime dimensions.

Note first that even before we take the long-distance limit, the elimination of the scalar mode of the graviton is certainly a good sign for the possible matching against general relativity at long distances, and so is the fact that the coupling constant $\lambda$ in the kinetic term is now frozen by the symmetries of the generally covariant theory to take the relativistic value $\lambda=1$. As a result, the number and the tensor structure of the gravitational wave polarizations is the same as in general relativity.

In the infrared limit of our theory, the potential $\mathcal{V}$ is dominated by the scalar curvature and the cosmological constant term, (1.18). In this regime, the natural scaling is isotropic, with dynamical exponent $z=1$. The low-energy physics is best represented in rescaled coordinates $\left(x^{0}, x^{i}\right)$ and in terms of rescaled fields. First, the new time coordinate

$$
x^{0}=\mu t
$$

is defined by absorbing the effective speed of light $\mu$ into the definition of time. Because $[\mu]=z-1$, this implies that $\left[x^{0}\right]=-1=\left[x^{i}\right]$, in accord with the $z=1$ scaling. The rescaled fields are defined by

$$
N_{i}^{\mathrm{IR}}=\frac{1}{\mu} N_{i}, \quad A^{\mathrm{IR}}=\frac{1}{\mu^{2}} A .
$$

This rescaling ensures (i) that $N_{i}^{\mathrm{IR}}$ carries the canonical dimension implied by $z=1$, and (ii) that the $U(1)$ gauge transformations are given by the standard relativistic formula

$$
\delta N_{i}^{\mathrm{IR}}=\partial_{i} \alpha^{\mathrm{IR}}, \quad \delta A^{\mathrm{IR}}=\partial_{0} \alpha^{\mathrm{IR}},
$$

with $\alpha^{\mathrm{IR}}=\alpha / \mu$. In the rest of the paper, we will drop the "IR" superscripts, and refer to the rescaled fields (5.2) in the infrared simply as $N_{i}$ and $A$.

The action of the infrared theory in the infrared variables is

$$
S_{\mathrm{IR}}=\frac{1}{16 \pi G_{N}} \int d x^{0} d^{D} \mathbf{x} \sqrt{g}\left\{N\left(K_{i j} K^{i j}-K^{2}+R-2 \Lambda\right)-A(R-2 \Omega)\right\}+\ldots,
$$

where "..." denotes corrections due to higher dimension operators, as well as the $\nu$-dependent terms in (4.21) which are unimportant for our arguments below. In (5.4), $K_{i j}$ refers to the extrinsic curvature tensor in the infrared coordinates, of canonical scaling dimension equal to one; and the Newton constant is given by

$$
G_{N}=\frac{\kappa^{2}}{32 \pi \mu}
$$


In the remainder of this section, we comment on three issues: The structure of compactobject solutions (which will be relevant for solar system tests), the issue of Lorentz symmetry, and the nature of cosmological solutions in the infrared regime of our theory as described by (5.4).

\subsection{Static compact-object solutions}

To prepare the ground for solar system tests, consider the infrared limit (5.4) and set the cosmological constant to zero. Interestingly, as the Schwarzschild black hole turns out to be a solution of this infrared theory. In terms of our fields, this solution will be represented by

$$
\begin{aligned}
& g_{i j} d x^{i} d x^{j}=\left(1-\frac{2 M}{r}\right)^{-1} d r^{2}+r^{2} d \Omega_{2}^{2}, \\
& \quad A=1-\left(1-\frac{2 M}{r}\right)^{1 / 2}, \quad N=1, \quad N_{i}=0, \quad \nu=0 .
\end{aligned}
$$

It is straightforward to see that this geometry satisfies the equations of motion of our theory for $\Omega=0$, which is the appropriate choice if we are interested in asymptotically flat solutions. First, the equations of motion contain the condition $R=2 \Omega$. With $\Omega=0$, this equation is indeed satisfied by the spatial slices (5.6) of the relativistic Schwarzschild metric in the Schwarzschild coordinate system. The $\nu$ and $N_{i}$ equations of motion are also satisfied, because the extrinsic curvature $K_{i j}$ vanishes for static backgrounds.

Finally, to show that the $g_{i j}$ equation of motion are also satisfied, we use a simple but intriguing argument. Since the same argument generalizes in a useful way to the case of nonzero $\Omega=\Lambda$, and also of arbitrary dimension, we present this more general case. Start with static solutions with $K_{i j}=0$, and observe that the equations of motion for $g_{i j}, N$ and $A$ are identical to the equations that follow from the following reduced action,

$$
\int d^{D} \mathbf{x} \sqrt{g}(N-A)(R-2 \Omega) .
$$

Similarly, for static solutions with $K_{i j}=0$ of general relativity in $D+1$ dimensions, the corresponding equations of motion are those of the reduced Einstein-Hilbert action,

$$
\int d^{D} \mathbf{x} \sqrt{g} \mathfrak{N}(R-2 \Lambda)
$$

where $\mathfrak{N}$ is the general-relativistic lapse function. Consequently, if we identify the $N$ and $A$ fields with the lapse function $\mathfrak{N}$ of general relativity,

$$
\mathfrak{N}=N-A,
$$

we see that static solutions of general relativity are also solutions of our theory in the infrared limit. In retrospect, this mapping also explains the form of $A$ in our representation of the Schwarzschild metric (5.7). 
Note that the relationship (5.10) between the general-relativistic lapse function $\mathfrak{N}$ and the $N$ and $A$ variables of our theory reproduces exactly what we would have expected from the geometric interpretation of $A$ as the subleading term in the expansion of the relativistic $g_{00}$ in powers of $1 / c$ as obtained in (3.6). Indeed, we start by expanding

$$
g_{00} \equiv-\mathfrak{N}^{2}=-(N-A)^{2} \approx-N^{2}+2 N A+\ldots
$$

Recall now that $A$ in (5.11) is the infrared rescaled field (5.2), related to the microscopic gauge field by a rescaling factor $1 / \mu^{2}$. Using the fact that $\mu$ plays the role of the speed of light (as we have seen in (5.1)), the two leading terms in (5.11) match exactly the leading two terms in the expansion (3.6).

These arguments prove that the Schwarzschild geometry in the Schwarzschild coordinates, with the indentification implied by (5.10), is a solution of the infrared limit of our theory, with $\Omega=\Lambda=0$. However, in the parametrized post-Newtonian (PPN) formalism [34,35] which is typically used in gravitational phenomenology, the compact-object solution is usually represented in the isotropic coordinates. In the case of general relativity, this is just a gauge choice, a fact which does not extend automatically to alternative approaches to gravity such as ours. Showing that the Schwarzschild geometry in the Schwarzschild coordinates is a solution of our theory does not imply that it will be a solution when represented in another coordinate system, because only those coordinate changes that belong to the gauge symmetry of our model will map a solution to a solution. However, because the transformation from the Schwarzschild coordinates to the isotropic ones only changes the radial coordinate,

$$
r=\rho\left(1+\frac{M}{2 \rho}\right)^{2},
$$

while keeping $t, \theta, \phi$ intact, it is a foliation-preserving diffeomorphism, a symmetry of the theory. Consequently, the Schwarzschild solution in the isotropic coordinates, represented by

$$
\begin{aligned}
g_{i j} d x^{i} d x^{j} & =\left(1+\frac{M}{2 \rho}\right)^{4}\left(d \rho^{2}+\rho^{2} d \Omega_{2}^{2}\right), \\
A & =\left(1+\frac{M}{2 \rho}\right)^{-1} \frac{M}{\rho}, \quad N=1, \quad N_{i}=0, \quad \nu=0,
\end{aligned}
$$

is also a solution of the infrared limit of our theory. Expanding this solution to the required order in the powers of $M / \rho$ strongly suggests that in the infrared regime, the $\beta$ and $\gamma$ parameters of the PPN formalism [34,35] will take the same values as in general relativity, $\beta=\gamma=1$. This feature is favorable for the solar-system tests of the theory.

\subsection{Lorentz symmetry}

Perhaps the leading challenge in any attempt to make theories of gravity with anisotropic scaling phenomenologically viable in $3+1$ dimensions is the issue of restoring Lorentz symmetry, at least at the intermediate energies and distances where it has been so well tested experimentally. In particular, we need a mechanism ensuring that in the corresponding regime, all 
species of matter (including the gravitons) percieve the same lightcones and the same effective speed of light. In the minimal theory with anisotropic scaling, this issue arises already for pure gravity: At generic values of the couplings, the speeds of the tensor and scalar graviton polarizations are not related by any symmetry, and are generally different from each other already in the short-distance regime. In contrast, our generally covariant theory has only the tensor graviton polarizations, all sharing the same speed at all energies; however, the issue reemerges when pure gravity is coupled to non-gravitational matter. If the present theory is to be phenomenologically viable, its coupling to matter will have to be analyzed in detail. This analysis is beyond the scope of the present paper; we only limit ourselves to one observation, which may be useful for the future analysis.

In general relativity, Lorentz symmetry is a global symmetry associated with the isometries of the Minkowski spacetime. In gravity with anisotropic scaling, we can adjust the couplings such that the flat spacetime geometry continues to be a solution. The global symmetries of this solution will then depend on the precise model of gravity with anisotropic scaling.

First consider the case of the minimal theory reviewed in Section (1.2), with the cosmological constant tuned to zero. The flat spacetime (2.1) is a solution, but it does not exhibit the full global Lorentz symmetry - the Lorentz boosts, generated by

$$
\delta t=b_{i} x^{i}, \quad \delta x^{i}=b_{i} t
$$

(with $b_{i}$ a constant vector), are not foliation-preserving diffeomorphisms. In this theory, the nonrelativistic analogs of the Killing symmetries of the flat spacetime solution correspond to spacetime translations and space rotations - the solution breaks all possible boost symmetries spontaneously, and defines a preferred rest frame.

In contrast, in our generally covariant theory, we can interpret the Lorentz transformation (5.15) as a generator of a transformation belonging to the extended symmetry group $U(1) \ltimes$ $\operatorname{Diff}(M, \mathcal{F})$. More precisely, the Lorentz transformation (5.15) should be interpreted as a composition of an infinitesimal foliation-preserving diffeomorphism and an infinitesimal $U(1)$ transformation. Indeed, restoring the factors of $c$ shows that the variation of $t$ in (5.15) is suppressed by a factor of $1 / c^{2}$ compared to the variation of $x^{i}$, and should therefore be interpreted as an infinitesimal $U(1)$ transformation with $\alpha=b_{i} x^{i}$, accompanied in (5.15) by the infinitesimal foliation-preserving diffeomophism

$$
\delta t=0, \quad \delta x^{i}=b_{i} t .
$$

When interpreted in this way, the Lorentz transformation (5.15) is a symmetry of the flat spacetime geometry represented in our variables by $g_{i j}=\delta_{i j}, N=1$ and $A=0$. This does not yet imply that all preferred-frame effects are absent in this background: In particular, the Newton prepotential $\nu$ is not invariant under the Lorentz boosts, and defines a preferred frame for the flat spacetime, in which $\nu=0$. The flat background is Lorentz invariant only to the extent that the effects of the Newton prepotential can be ignored. 


\subsection{Cosmological solutions}

Moving beyond asymptotically flat spacetimes, it is natural to ask whether our theory has interesting cosmological solutions. One can start with a given spacetime geometry in general relativity, and investigate whether it satisfies the equations of motion of our theory. The answer to this question will again depend on the choice of spacetime foliation.

For example, arguments identical to those used above for the Schwarzschild metric show that the static patch of the de Sitter (or anti-de Sitter) spacetime, represented in our variables by

$$
\begin{aligned}
& g_{i j} d x^{i} d x^{j}=\left(1-\frac{\Lambda r^{2}}{3}\right)^{-1} d r^{2}+r^{2} d \Omega_{2}^{2}, \\
& A=1-\left(1-\frac{\Lambda r^{2}}{3}\right)^{1 / 2}, \quad N=1, \quad N_{i}=0, \quad \nu=0
\end{aligned}
$$

is a solution of our theory if we set $\Omega=\Lambda$.

It is encouraging to see that at least in the time-independent foliations, the de Sitter and anti-de Sitter spacetimes are solutions of our theory. In standard cosmological applications, however, the cosmological principle selects another natural foliation of spacetime, with homogeneous spatial slices and a time-dependent scale factor $a(t)$. On the face of it, it may appear difficult to obtain cosmological solutions of our theory with maximally symmetric and timedependent spatial slices: The equation of motion for $A$ plays the role of a Gauss constraint, and implies $R=2 \Omega$ in the vacuum. Assuming the standard FRW Ansatz

$$
g_{i j}=a^{2}(t) \gamma_{i j}, \quad N=1, \quad A=0, \quad N_{i}=0, \quad \nu=0
$$

where $\gamma_{i j}$ is a time-independent maximally symmetric spatial metric, the scalar curvature of $g_{i j}$ has to be constant in time. Consequently, if the scalar curvature of $\gamma_{i j}$ is nonzero, the cosmological scale factor must be independent of time.

Of course, if our spatial slices are flat, the Gauss constraint no longer restricts the time dependence of the cosmological scale factor. This requires $\Omega=0$. The rest of the equations of motion will be satisfied by the de Sitter spacetime in the inflationary coordinates, which in the FRW Ansatz (5.19) corresponds to

$$
a(t)=e^{H t}, \quad \gamma_{i j}=\delta_{i j}
$$

The reason for this is again simple but illuminating: With $\Omega=0$, the $\nu$ equation of motion is satisfied when the metric is flat. With $\nu$ and $A$ both zero, the remaining equations are implied by Einstein's equations if we simply identify the relativistic lapse function with our $N(t)$, the relativistic cosmological constant with our $\Lambda$, and $H$ with the Hubble constant.

Thus, we see that the same de Sitter spacetime in two different foliations is a solution of the infrared theory for two different choices of the coupling constant, one with $\Omega=\Lambda$ and the other with $\Omega=0$ and nonzero $\Lambda$. Mapping out the general behavior of cosmological solutions 
as the coupling constants $\Omega$ and $\Lambda$ are independently varied is one of questions left for future work.

In addition, there are at least two ways out of the potential difficulty with solving the Gauss constraint for cosmologically evolving spacetimes with maximally symmetric spatial slices of nonzero curvature. First, the equations of motion will change in the presence of matter. In the full system of equations for gravity and matter, the Gauss constraint is expected to be modified by a matter source, whose time dependence can then drive the time dependence of the scale factor in the spatial metric. The second possibility is related to the gauge freedom we have in describing cosmological solutions in general relativity: Instead of the standard FRW ansatz which leads to (5.19), one can choose coordinates in which the spatial metric is not only maximally symmetric but also constant in time. When we express the FRW geometry in such coordinates, the time-dependent scale factor appears in the $d t^{2}$ term in the metric, and non-zero components of the shift vector $N_{i}$ are typically generated. In general relativity, this coordinate representation of FRW cosmologies is a legitimate albeit slightly unconventional gauge choice. In our theory, this parametrization of FRW universes has the advantage of being compatible with the vacuum Gauss constraint $R=2 \Omega$.

\section{Acknowledgments}

We wish to express our thanks to Niayesh Afshordi, Jan Ambjørn, Dario Benedetti, Diego Blas, Robert Brandenberger, Gia Dvali, Marc Henneaux, Elias Kiritsis, Renate Loll, Alex Maloney, Shinji Mukohyama, Yu Nakayama, Oriol Pujolàs, Sergey Sibiryakov, Arkady Vainshtein, and the participants of the Perimeter Institute workshop on Gravity at a Lifshitz Point (November 2009) for useful discussions. P.H. is grateful to the Arnold Sommerfeld Center for Theoretical Physics, Ludwig-Maximilians-Universität, München, and the PH-TH Division, CERN, Genève, for their hospitality during some of the final stages of this work. The results of this work were presented at the GR 19 Conference in Mexico City in July 2010; P.H. wishes to thank the organizers for their invitation and hospitality. This work has

been supported by NSF Grant PHY-0855653, DOE Grant DE-AC02-05CH11231, and by the Berkeley Center for Theoretical Physics.

\section{References}

[1] P. Hořava, Membranes at Quantum Criticality, JHEP 03 (2009) 020, arXiv:0812.4287.

[2] P. Hořava, Quantum Gravity at a Lifshitz Point, Phys. Rev. D79 (2009) 084008, arXiv:0901.3775.

[3] P. Hořava, Spectral Dimension of the Universe in Quantum Gravity at a Lifshitz Point, Phys. Rev. Lett. 102 (2009) 161301, arXiv:0902.3657].

[4] R. L. Arnowitt, S. Deser, and C. W. Misner, The Dynamics of General Relativity, in Gravitation: an Introduction to Current Research (Wiley 1962), gr-qc/0405109. 
[5] N. Afshordi, Cuscuton and Low Energy Limit of Hořava-Lifshitz Gravity, Phys. Rev. D80 (2009) 081502, arXiv:0907.5201.

[6] S. Dutta and E. N. Saridakis, Overall Observational Constraints on the Running Parameter $\lambda$ of Horava-Lifshitz Gravity, JCAP 1005 (2010) 013, arXiv:1002.3373.

[7] C. Charmousis, G. Niz, A. Padilla, and P. M. Saffin, Strong Coupling in Hořava Gravity, JHEP 08 (2009) 070, arXiv:0905.2579.

[8] D. Blas, O. Pujolàs, and S. Sibiryakov, On the Extra Mode and Inconsistency of Hor̆ava Gravity, JHEP 10 (2009) 029, arXiv:0906.3046.

[9] D. Blas, O. Pujolàs, and S. Sibiryakov, Consistent Extension of Hor̆ava Gravity, Phys. Rev. Lett. 104 (2010) 181302, arXiv:0909.3525.

[10] M. Henneaux and C. Teitelboim, Quantization of Gauge Systems. Princeton U.P., 1992.

[11] H. Ruegg and M. Ruiz-Altaba, The Stueckelberg Field, Int. J. Mod. Phys. A19 (2004) 3265-3348, hep-th/0304245.

[12] C. Germani, A. Kehagias, and K. Sfetsos, Relativistic Quantum Gravity at a Lifshitz Point, JHEP 09 (2009) 060, arXiv:0906.1201.

[13] I. Kimpton and A. Padilla, Lessons from the Decoupling Limit of Hor̆ava Gravity, JHEP 07 (2010) 014, arXiv:1003.5666.

[14] C. Teitelboim, The Hamiltonian Structure of Space-Time, in: General Relativity and Gravitation, Vol.1 (Plenum Press, 1980) ed: A. Held.

[15] M. Henneaux, Zero Hamiltonian Signature Spacetimes, Bull. Soc. Math. Belg. 31 (1979) 47.

[16] P. A. M. Dirac, Generalized Hamiltonian Dynamics, Proc. Roy. Soc. Lond. A246 (1958) $326-332$.

[17] P. A. M. Dirac, The Theory of Gravitation in Hamiltonian Form, Proc. Roy. Soc. Lond. A246 (1958) 333-343.

[18] J. Klusoň, Note about Hamiltonian Formalism of Healthy Extended Hořava-Lifshitz Gravity, arXiv: 1004.3428.

[19] M. Henneaux, A. Kleinschmidt, and G. L. Gomez, A Dynamical Inconsistency of Hořava Gravity, Phys. Rev. D81 (2010) 064002, arXiv:0912.0399.

[20] P. Hořava, Quantum Gravity at a Lifshitz Point. review talk at Strings 2009, Rome, June 2009.

[21] M. Li and Y. Pang, A Trouble with Hořava-Lifshitz Gravity, JHEP 08 (2009) 015, arXiv:0905.2751.

[22] S. Farkas and E. J. Martinec, Gravity from the Extension of Spatial Diffeomorphisms, arXiv: 1002.4449 .

[23] J. Bellorín and A. Restuccia, On the Consistency of the Hor̆ava Theory, arXiv:1004.0055.

[24] J. M. Pons and P. Talavera, Remarks on the Consistency of Minimal Deviations from General Relativity, Phys. Rev. D82 (2010) 044011, arXiv:1003.3811.

[25] E. Witten, Topological Quantum Field Theory, Commun. Math. Phys. 117 (1988) 353. 
[26] E. Witten, Introduction to Cohomological Field Theories, Int. J. Mod. Phys. A6 (1991) $2775-2792$.

[27] W. Li, W. Song, and A. Strominger, Chiral Gravity in Three Dimensions, JHEP 04 (2008) 082, arXiv:0801.4566.

[28] A. Maloney, W. Song, and A. Strominger, Chiral Gravity, Log Gravity and Extremal CFT, Phys. Rev. D81 (2010) 064007, arXiv:0903.4573.

[29] R. M. Wald, Spin-2 Fields and General Covariance, Phys. Rev. D33 (1986) 3613.

[30] C. Xu and P. Hořava, Emergent Gravity at a Lifshitz Point from a Bose Liquid on the Lattice, Phys. Rev. D81 (2010) 104033, arXiv:1003.0009.

[31] P. Hořava and E. Witten, Heterotic and Type I String Dynamics from Eleven Dimensions, Nucl. Phys. B460 (1996) 506, hep-th/9510209.

[32] P. Hořava and E. Witten, Eleven-Dimensional Supergravity on a Manifold with Boundary, Nucl. Phys. B475 (1996) 94-114, hep-th/9603142.

[33] P. Hořava and C. M. Melby-Thompson, Anisotropic Conformal Infinity, arXiv:0909.3841.

[34] C. M. Will, Theory and Experiment in Gravitational Physics. Cambridge University Press, 1993 (2nd ed.).

[35] C. M. Will, The Confrontation Between General Relativity and Experiment, Living Rev. Rel. 9 (2005) 3, gr-qc/0510072. 